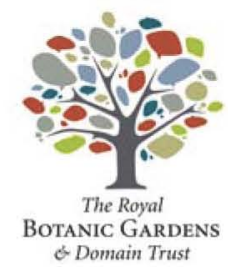

\title{
A REVISION OF GALIUM (RUBIACEAE) IN AUSTRALIA AND NEW ZEALAND
}

\author{
D.J. McGillivray \\ (Accepted for publication 26.6.1981)
}

\begin{abstract}
McGillivray, D.J. (National Herbarium of New South Wales, Royal Botanic Gardens, Sydney, Australia 2000) 1983. A revision of Galium (Rubiaceae) in Australia and New Zealand. Telopea 2(4): 355-377. - Twelve native species are described including the new species G. terraereginae Ehrendorfer et McGillivray (Queensland, p.361), G. compactum Ehrendorfer et McGillivray (Victoria, South Australia; p.370), G. roddil Ehrendorfer et McGillivray (New South Wales, p.371), and G. curvihirtum Ehrendorfer et McGillivray (Victoria, South Australia; p.373). The new name G. migrans Ehrendorfer et McGillivray (p.362) is provided for G. erythrorrhizum F. Muell. ex Miq. non Boiss. et Reut., and G. trilobum Colenso replaces G. tenuicaule A. Cunn. non Krocker. A key to the species is provided on p.356; non-indigenous species are included only in the key.
\end{abstract}

\section{INTRODUCTION}

The earliest collections of Galium from New Zealand and Australia were made in 1769 by Banks and Solander on the coast of New Zealand. Solander's manuscript name for this species was incidentally mentioned by G. Forster (1786: 89), but the first names to be validly published, $G$. gaudichaudii DC. and G. australe DC., did not appear until 1830. The former species was described from material collected in 1819 by Gaudichaud during Freycinet's voyage (1817-1820); G. australe was named from specimens gathered in 1827 during the voyage of the "Astrolabe" (1826-1829) under the command of J.S.C. Dumont d'Urville. Allan Cunningham (1839: 205) described the two New Zealand species from collections made by his brother Richard, thus providing a valid name, $G$. propinquum, to replace Solander's manuscript name, and a later homonym, $G$. tenuicaule, for the species which is now known as $G$. trilobum Colenso. Further contributions to the taxonomy of Galium in Australia and New Zealand were made by J.D. Hooker (1847: 461 bis, 462 bis, 1852: 113), Miquel (1856: 113), Bentham (1867: 445), F. Mueller (1875: 188), Colenso (1888: 192), Ewart and Rees (1913: 4), Black (1929: 538), Ewart (1931: 1047), and Wakefield (1955: 69).

Bentham made the only comprehensive treatment of the genus in Australia. $\mathrm{He}$ recognized five indigenous species, of which three are upheld in this revision. Some changes in their delimitations have been necessary. Wakefield made the next significant improvement by recognizing two new species but at the same time retaining distinct species within $G$. gaudichaudii and $G$. propinquum. From the more ample material now available it is evident that Wakefield and earlier workers were hampered by the lack of adequate collections.

This paper was prepared with the assistance and guidance of Professor F. Ehrendorfer (University of Vienna) who has been interested in the Rubiaceae since the nineteen-forties and has made various cytological, evolutionary and taxonomic studies, particularly in the Galieae. During 1966 he visited Australia on a Senior Research Fellowship from the Australian Academy of Science. His studies of the collections of Australian Galieae in Herbarium Australiense (CANB) and his observations in the field revealed taxa which had not been recognised in the existing classifications of Australian Galia. At about the same time my own studies of the collections in the National Herbarium of New South Wales (NSW) indicated some of the inadequacies which had been independently discovered by Ehrendorfer. 
Discussions between us about the delimitation of taxa led to the development of this study. Professor Ehrendorfer gave me further valuable assistance with the paper during my visit to Graz (Austria) in July 1969. In a future paper Ehrendorfer proposes to discuss the affinities of the Australian and New Zealand species and to deal with broader aspects of their classification.

Although living plants were studied, the descriptions are based entirely on dried specimens. All of the specimens cited were examined unless otherwise indicated.

Comparative tables of selected features for each species are included after the key; see Tables 1, 2 (pp. 358-360).

\section{KEY TO SPECIES}

(To assist users in retracing their steps through the key the number of the preceding lead is given in parenthesis.)

A. Leaves and stipules in whorls of 4(-5); if in whorls of 5 then leaves usually $10-15 \mathrm{~mm}$ long, 2.5-5 mm wide, and fruits ribbed. Perennials and ? annuals. Indigenous species. (for lead B: non-indigenous species-see p.358)

1 Ovaries and fruits smooth, papillose, rugose or ribbed, never tuberculate or hairy $\ldots \ldots 2$

1* Ovaries and fruits tuberculate or hairy $\ldots \ldots \ldots \ldots \ldots \ldots \ldots \ldots \ldots \ldots \ldots \ldots \ldots \ldots$

2 Cymes equal to or shorter than the leaves $\ldots \ldots \ldots \ldots \ldots \ldots \ldots \ldots \ldots \ldots \ldots \ldots$

2* Cymes longer than the leaves $\ldots \ldots \ldots \ldots \ldots \ldots \ldots \ldots \ldots \ldots \ldots \ldots \ldots \ldots \ldots \ldots$

3(2) Longest pedicel in each cyme more than $2 \mathrm{~mm}$ in length $\ldots \ldots \ldots \ldots \ldots \ldots \ldots$

$3^{*} \quad$ Longest pedicel in each cyme $2 \mathrm{~mm}$ or less in length $\ldots \ldots \ldots \ldots \ldots \ldots$

4(3) Leaf-base abruptly rounded to a short petiole, upper surface of leaf glossy; internodes

(6-) $8-18(-25) \mathrm{mm}$ long; flowers often yellowish $\ldots \ldots \ldots \ldots \ldots \ldots \ldots \ldots \ldots \ldots$ ciliare

4* Leaf-base tapering or gradually rounded to a short petiole, upper surface of leaf matt; internodes (3-) $10-30(-45) \mathrm{mm}$ long; flowers cream to white $\ldots \ldots \ldots \ldots \ldots$. propinquum

$5\left(3^{*}\right) \quad$ All stems prostrate to procumbent; leaves more than $2 \mathrm{~mm}$ wide; fruits prominently

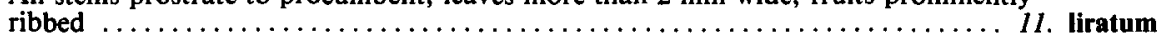

5* Some stems erect or ascending; leaves usually less than $2 \mathrm{~mm}$ wide; fruits rugose to

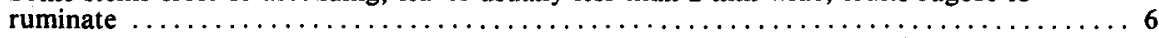

6(5*) Rhizomes absent or sparingly developed; internodes (5-) 12-20 (-35) mm long, leaves narrowly ovate to linear, (4-) $5-8(-12) \mathrm{mm}$ long, often irregularly curved in drying

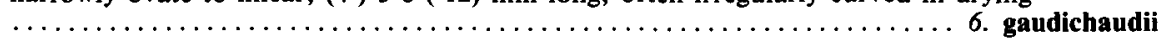

6* Rhizomes usually evident; internodes (2-) 3-8 (-15) $\mathrm{mm}$ long; leaves ovate to narrowly ovate, (2.5-) 3.5-4.5 (-6) mm long, not irregularly curved in drying ...... 7. compactum

$7\left(2^{*}\right) \quad$ Leaf-base abruptly rounded to a short petiole, upper surface of leaf glossy; internodes (6-) 8-18 (-25) $\mathrm{mm}$ long; flowers often yellowish $\ldots \ldots \ldots \ldots \ldots \ldots \ldots \ldots \ldots \ldots$. ciliare

7* Leaf-base tapering or gradually rounded to a short petiole, or non-petiolate, upper surface of leaf matt; internodes (5-) $10-40(-80) \mathrm{mm}$ long; flowers cream to white $\ldots \ldots .8$

$8\left(7^{*}\right)$ On lower parts of stems the pairs of leaves and stipules very unequal in length (leaf length: stipule length $>3: 2$ ); stems retrorsely aculeate or smooth, indumentum never conspicuous; leaf length:breadth $>3: 1 \ldots \ldots \ldots \ldots \ldots \ldots \ldots \ldots \ldots \ldots$ binifolium

8* On lower parts of stems the pairs of leaves and stipules slightly unequal (leaf length: stipule length $<3: 2$ ); leaf-ratio and indumentum of stems various $\ldots \ldots \ldots \ldots \ldots \ldots$

$9\left(8^{*}\right)$ Primary root conspicuous and/or rhizomes absent or sparingly developed; lower parts of stems comparatively stiff and brittle; inflorescences (2-) 3-8 (-25)-flowered

9* Primary root usually not evident, rhizomes usually well-developed; stems comparatively weak and flexible throughout; inflorescences $1-5$ (-7) commonly 3 ,-flowered 
10(9) Leaf margin recurved to almost flat; pedicels (1-) 2-3.5 (-8) mm long (leaves almost

flat and straight when dried) $\ldots \ldots \ldots \ldots \ldots \ldots \ldots \ldots \ldots \ldots \ldots \ldots \ldots \ldots, \ldots \ldots \ldots$, migrans

10* Leaf margin strongly recurved; pedicels $0.1-1(-2) \mathrm{mm}$ long (leaves commonly irregularly

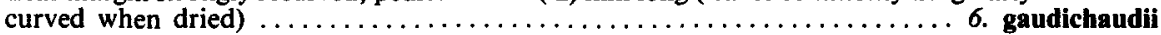

$11\left(9^{*}\right)$ Peduncles curved, longer than the pedicels; leaves narrowly obovate to elliptical; leaf length:breadth $>3: 1 \ldots \ldots \ldots \ldots \ldots \ldots \ldots \ldots \ldots \ldots \ldots \ldots \ldots \ldots \ldots \ldots \ldots$, trilobum

11* Peduncles straight (or sometimes absent), shorter than the pedicels; leaves ovate to narrowly ovate or elliptical; leaf length:breadth usually $3: 1$ or less $\ldots \ldots \ldots 4$. propinquum

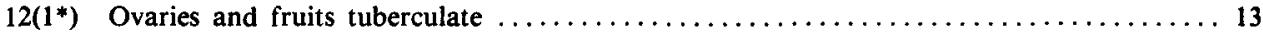

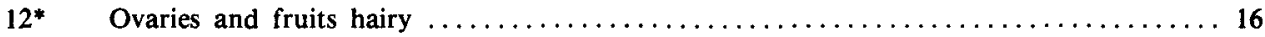

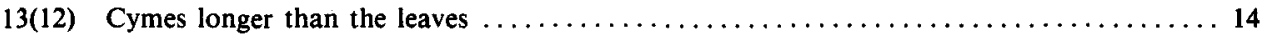

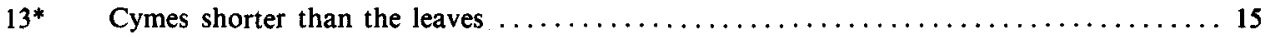

14(13) Primary root conspicuous and/or rhizomes absent or sparingly developed; lower parts of stems comparatively stiff and brittle; inflorescences (2-) 3-8 (-25)-flowered, peduncles

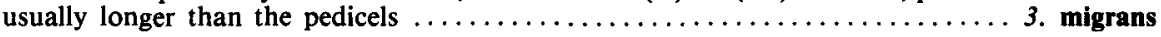

14* Primary root usually not evident, rhizomes usually well-developed; stems comparatively weak and flexible throughout; inflorescences $1-5(-7)-$, commonly 3 , -flowered, peduncles shorter than the pedicels $\ldots \ldots \ldots \ldots \ldots \ldots \ldots \ldots \ldots \ldots \ldots \ldots \ldots \ldots \ldots \ldots \ldots \ldots \ldots$, propinquum

15(13*) Longest pedicel of each cyme less than $1 \mathrm{~mm}$ long; fructual $\dagger$ tubercles rounded $\ldots 8$. roddii

15* Longest pedicel of each cyme $2 \mathrm{~mm}$ or more in length; fructual $\dagger$ tubercles pointed

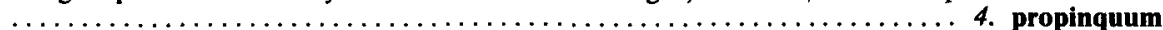

16(12*) Fructual $\dagger$ hairs each with a conspicuous terminal hook, hairs spreading, their length c. equal to or more than half the width of each mericarp $\ldots \ldots \ldots \ldots \ldots \ldots$, . australe

16* Fructual $\dagger$ hairs straight or curved, or if conspicuously hooked then their length noticeably

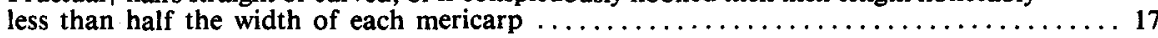

$17\left(16^{*}\right)$ On lower parts of stems the pairs of leaves and stipules very unequal in length (leaf length: stipule length $>3: 2$ ); peduncles longer than pedicels; leaf length:breadth $>3: 1.9$. binifolium

17* On lower parts of stems the pairs of leaves and stipules slightly unequal (leaf length: stipule length $<3: 2$ ); peduncle:pedicel ratio and leaf-ratio various $\ldots \ldots \ldots \ldots \ldots \ldots \ldots$

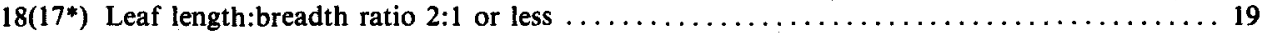

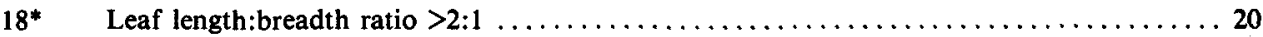

19(18) Stems comparatively weak and flexible, commonly glabrous to retrorsely aculeate; pedicels straight or uniformly curved $\ldots \ldots \ldots \ldots \ldots \ldots \ldots \ldots \ldots \ldots \ldots \ldots \ldots \ldots \ldots$. propinquum

19* Stems comparatively stiff and brittle, densely covered with spreading hairs; pedicels noticeably curved at their apices $\ldots \ldots \ldots \ldots \ldots \ldots \ldots \ldots \ldots \ldots \ldots \ldots \ldots \ldots$, terrae-reginae

20(18*) Peduncles shorter than pedicels; stems comparatively weak and flexible throughout; primary root usually not evident, rhizomes usually well-developed .......4. propinquum

$20^{*} \quad$ Peduncles longer than pedicels; lower parts of stems comparatively stiff and brittle; primary roots conspicuous and/or rhizomes absent or sparingly developed $\ldots \ldots \ldots \ldots, 2$

21(20*) Pedicels 0.5-1.5 (-2.5) mm long; flowers (1-) 2-3 (-5) per peduncle; fruits densely covered with short curved usually whitish hairs $\ldots \ldots \ldots \ldots \ldots \ldots \ldots \ldots \ldots \ldots 10$. curvihirtum

21* Pedicels (1-) 2-4 (-8) mm long; flowers (2-) 3-7 (-25) per peduncle; fructual indumentum variable, sometimes similar to that of $G$. curvihirtum but not so short and close 3. migrans

†Fructual, an adjective derived from fructus, comparable to staminal, and modelled on other nouns of the fourth declension. The new word may add flexibility to descriptions of fruits. 
B.(A) Leaves and stipules in whorls of (4-) 6-9, if in whorls of 4-6 then leaves usually 3-8 $\mathrm{mm}$ long and fruits narrowly oblong with hooked hairs. Annuals. Non-indigenous species (for descriptions of these species see Tutin et al., Fl. Europaea 4: 14-36 [1976].)

1 Stems comparatively robust usually $>50 \mathrm{~cm}$ long; leaf margin with retrorse hairs $\ldots \ldots \ldots 2$

1* Stems comparatively delicate usually $<25 \mathrm{~cm}$ long; leaf margin with antrorse hairs $\ldots \ldots . .3$

2 Fruits with hooked hairs; upper surface of leaves hairy ........... G. aparine L.

$2^{*} \quad$ Fruits with numerous tubercles or papillae; upper surface of leaves glabrous G. tricornutum Dandy

3 Fruits with hooked hairs; inflorescences few-flowered G. murale (L.) All.

3* Fruits papillose; inflorescences many-flowered

G. divaricatum Pourret ex Lamk.

Table 1. Comparison of selected vegetative features

\begin{tabular}{llll} 
Species & Root System & $\begin{array}{l}\text { Internode length (mm) } \\
\text { and stem indumentum }\end{array}$ & $\begin{array}{l}\text { Leaf shape } \\
\text { and size (mm) }\end{array}$ \\
\hline 1. australe & $\begin{array}{l}\text { strong primary root } \\
\text { and nodal roots up to } \\
15 \mathrm{~cm} \text { from rootstock }\end{array}$ & $\begin{array}{l}(8-) 15-45(-90) \\
\text { retrorsely aculeate, } \\
\text { sparsely to densely } \\
\text { covered with recurved or } \\
\text { spreading hairs or rarely } \\
\text { glabrous }\end{array}$ & $\begin{array}{l}\text { elliptical to narrowly } \\
\text { ovate or oblong (4-) } \\
6-12(-18) \times 1.5-4(-6)\end{array}$ \\
& &
\end{tabular}

\section{2. terrae-reginae}

strong primary root with nodal roots up to $5 \mathrm{~cm}$ from the rootstock

3. migrans

strong primary root and nodal roots up to $20 \mathrm{~cm}$ from the rootstock

4. propinquum

primary root later supplemented or superseded by nodal roots from welldeveloped slender rhizomes

5. ciliare

6. gaudichaudii

7. compactum

8. roddii

nodal roots from subterranean stems, primary root not conspicuous weak development of nodal roots up to $5 \mathrm{~cm}$ from the rootstock

\section{(4) $8-20(-25)$} numerous spreading hairs

(5-) $15-40$ (-100) commonly retrorsely aculeate, sometimes with many to few spreading to retrorse hairs, rarely glabrous

(3-) 10-30 (-45) commonly retrorsely aculeate to glabrous, or or with spreading to retrorse hairs

(6-) 8-18 (-25)

spreading dense to sparse hairs or glabrous, not retrorsely aculeate

strong primary root, (5-) 12-20 (-35) hirsute, retrorsely aculeate or sometimes almost glabrous

(2-) 3-8 (-15) pilose to almost glabrous, not retrorsely aculeate ovate, narrowly ovate or elliptical (2.5-) 3-5 $(-7) \times(1.5-)$ 2-2.5 (-3.5)

elliptical to ovate, sometimes almost linear (3-) 5-10 (-18) $\mathrm{x}(1-)$ 1.5-3 (-5)

elliptical to narrowly ovate or ovate, sometimes almost round (1.5-) 2-8 (-15) $x(0.7-) 1-2.5(-6)$

ovate, sometimes rotund (2.5-) 3.5-8 (-10) $x(1-) 1.5-3(-4)$

narrowly ovate to linear, often almost narrowly triangular (4-) 5-8 (12) $\mathrm{x}$ (0.6-) $0.8-1.2(-2.0)$

ovate to narrowly ovate (2.5-) $3.5-4.5(-6) \times(0.8-)$ $1.5-1.8(-2.2)$

Notes 


\begin{tabular}{|c|c|c|c|c|}
\hline 9. binifolium & $\begin{array}{l}\text { strong to rather weak } \\
\text { primary roots some- } \\
\text { times supplemented } \\
\text { by occasional nodal } \\
\text { roots }\end{array}$ & $\begin{array}{l}(20-)) 30-60(-80) \\
\text { retrorsely aculeate } \\
\text { or smooth, lacking } \\
\text { a conspicuous } \\
\text { indumentum }\end{array}$ & $\begin{array}{l}\text { narrowly elliptical, } \\
\text { commonly slightiy falcate } \\
(4-) 6-12(-20) \times(0.7-) \\
1-2.5(-3)\end{array}$ & $\begin{array}{l}\text { leaves often } \\
\text { reclinate to } \\
\text { retrorse; foliaceous } \\
\text { stipules noticeably } \\
\text { shorter than leaves }\end{array}$ \\
\hline 10. curvihirtum & $\begin{array}{l}\text { primary root well- } \\
\text { developed, nodal } \\
\text { roots absent }\end{array}$ & $\begin{array}{l}\text { (10-) } 15-35(-60) \\
\text { commonly retrorsely } \\
\text { aculeate sometimes with } \\
\text { spreading or retrorse } \\
\text { hairs or glabrous }\end{array}$ & $\begin{array}{l}\text { narrowly ovate-linear, } \\
\text { sometimes ovate or } \\
\text { elliptical (3-) } 4-12(-15) \\
\times(0.6-) 1-2(2.5)\end{array}$ & \\
\hline 11. liratum & $\begin{array}{l}\text { primary root, not } \\
\text { always strongly } \\
\text { developed, nodal } \\
\text { roots rare }\end{array}$ & $\begin{array}{l}30-50 \\
\text { retrorsely aculeate to } \\
\text { glabrous }\end{array}$ & $\begin{array}{l}\text { obovate to elliptical } \\
(6-) 10-15(-20) \times(1.5-) \\
2.5-5(-7)\end{array}$ & $\begin{array}{l}\text { leaves usually } \\
\text { broader than those } \\
\text { of other species }\end{array}$ \\
\hline 12. trilobum & $\begin{array}{l}\text { nodal roots (primary } \\
\text { roots not observed) }\end{array}$ & $\begin{array}{l}(15-) 25-40(-50) \\
\text { retrorsely aculeate or } \\
\text { sometimes almost } \\
\text { smooth }\end{array}$ & $\begin{array}{l}\text { narrowly obovate to } \\
\text { elliptical, sometimes sub- } \\
\text { linear to spathulate (4-) } \\
6-12(-20) \times 1.5-3(-4)\end{array}$ & \\
\hline
\end{tabular}

Table 2. Selected features of inflorescences and fruits; distribution

\begin{tabular}{|c|c|c|c|c|}
\hline Species & Ultimate Peduncles & Pedicels (mm) & Fructual Surface & Distribution* \\
\hline 1. australe & $\begin{array}{l}1-7(-10) \text {-flowered }(0-) \\
5-12(-25) \mathrm{mm} \text { long } \\
\text { straight }\end{array}$ & $\begin{array}{l}\text { (0.5-) } 2.5-10(-22) \text { slightly } \\
\text { or strongly curved usually } \\
\text { within } 2 \mathrm{~mm} \text { of ovary or } \\
\text { fruit, rarely straight }\end{array}$ & $\begin{array}{l}\text { with numerous } \\
\text { usually transparent, } \\
\text { uncinate hairs } \\
0.4-1.0 \mathrm{~mm} \text { long }\end{array}$ & $\begin{array}{l}\text { NSW, VIC (E. of } \\
146^{\circ} \text { E), TAS, SA } \\
\text { (Kangaroo Island only) } \\
\text { - see fig. 1. }\end{array}$ \\
\hline 2. terrae-reginae & $\begin{array}{l}\text { 1-3-flowered } \\
1-5(-7) \mathrm{mm} \text { long } \\
\text { straight }\end{array}$ & $\begin{array}{l}1-3(-5) \text { conspicuously } \\
\text { curved near apex } \\
\text { particularly at the } \\
\text { fruiting stage }\end{array}$ & $\begin{array}{l}\text { tuberculate to rugose } \\
\text { and covered with } \\
\text { numerous slightly to } \\
\text { strongly curved hairs }\end{array}$ & Q-see fig. 1 \\
\hline 3. migrans & $\begin{array}{l}\text { (2-) } 3-7(-25)-\text { flowered } \\
\text { (2-) } 5-15(-35) \mathrm{mm} \\
\text { long } \\
\text { straight }\end{array}$ & $\begin{array}{l}\text { (1-) } 2-3.5(-8) \text { usually } \\
\text { slightly curved }\end{array}$ & $\begin{array}{l}\text { exceedingly variable: } \\
\text { papillose, rugose, } \\
\text { tuberculate or covered } \\
\text { with curved to straight } \\
\text { aculei or hairs }\end{array}$ & $\begin{array}{l}\text { Q, NSW (incl. ACT), } \\
\text { VIC, SA, WA-see } \\
\text { fig. } 1 .\end{array}$ \\
\hline 4. propinquum & $\begin{array}{l}\text { 1-5 (-7)-flowered, } \\
\text { often 3-flowered, } \\
\text { often absent or } \\
\text { inconspicuous, } \\
\text { maximum length } 6 \mathrm{~mm} \\
\text { usually straight }\end{array}$ & $\begin{array}{l}(0.3-) 1-4(-7) \\
\text { often curved }\end{array}$ & $\begin{array}{l}\text { papillose, tuberculate } \\
\text { (with pointed } \\
\text { tubercles), ruminate, } \\
\text { rugose or with } \\
\text { curved ascending } \\
\text { hairs, sometimes } \\
\text { exhibiting a } \\
\text { combination of these }\end{array}$ & $\begin{array}{l}\text { NZ, Q, NSW, VIC, } \\
\text { SA-see fig. } 2\end{array}$ \\
\hline 5. ciliare & $\begin{array}{l}\text { 5-9-flowered } \\
\text { 5-15 mm long } \\
\text { straight }\end{array}$ & $1-5$ straight & $\begin{array}{l}\text { papillose but } \\
\text { otherwise smooth }\end{array}$ & $\begin{array}{l}\text { Q, NSW (incl. ACT), } \\
\text { VIC, TAS-see fig. } 3\end{array}$ \\
\hline 6. gaudichaudii & $\begin{array}{l}\text { 1-5 (-7)-flowered } \\
\text { up to } 2.5 \mathrm{~mm} \text { long } \\
\text { straight }\end{array}$ & $\begin{array}{l}0.1-1.0(-2.0) \\
\text { straight }\end{array}$ & $\begin{array}{l}\text { rugulose, without } \\
\text { tubercles or hairs }\end{array}$ & $\begin{array}{l}\text { Q, NSW (incl. ACT), } \\
\text { VIC, TAS, SA- } \\
\text { see fig. } 4\end{array}$ \\
\hline 7. compactum & $\begin{array}{l}1-2(-3) \text {-flowered } \\
\leqslant 0.5 \mathrm{~mm} \text { long }\end{array}$ & up to c. 0.5 & rugose & VIC, SA-see fig. 3 \\
\hline 8. roddii & $\begin{array}{l}1-2(-3) \text {-flowered } \\
0.3-0.5 \mathrm{~mm} \text { long }\end{array}$ & $0.3-0.5$ & $\begin{array}{l}\text { covered with many } \\
\text { rounded pale-capped } \\
\text { tubercles }\end{array}$ & NSW-see fig. 3 \\
\hline 9. binifolium & $\begin{array}{l}\text { often 3-flowered, } \\
\text { up to 8-flowered } \\
\text { (2-) 4-25 mm long } \\
\text { straight to slightly } \\
\text { curved }\end{array}$ & $\begin{array}{l}2-5(-7) \\
\text { straight }\end{array}$ & $\begin{array}{l}\text { rugose or sometimes } \\
\text { ribbed, glabrous or } \\
\text { rarely with numerous } \\
\text { curved short hairs }\end{array}$ & $\begin{array}{l}\text { NSW, VIC, SA- } \\
\text { see fig. } 5\end{array}$ \\
\hline
\end{tabular}


10. curvihirtum

(1-) 2-3 (-5)-flowered (0.5-) $1.6(-11) \mathrm{mm}$ long

straight

11. liratum

12. trilobum

\section{1-3 (-5)-flowered} $\leqslant 1 \mathrm{~mm}$ long
$0.5-1.5(-2.5)$ straight or slightly curved

(1-) 2-3-flowered, (5-) (1-) 3-6 (-10) usually $8-14(-25) \mathrm{mm}$ long curved curved densely covered with VIC, SA-see fig. 5 curled whitish hairs (finer and shorter than those of Galium australe)

ribbed, with 5-7 irregular longitudinal ribs

finely ruminate to $\mathrm{NZ}$-see fig. 7 smooth

* NZ-New Zealand; Q-Queensland, NSW-New South Wales, ACT-Australian Capital Territory, VIC-Victoria, TAS-Tasmania, SA-South Australia, WA-Western Australia.

1. Galium australe $D C$., Prodr. 4: 608 (1830) non Reiche (1900); J.D. Hooker, Fl. Tasmaniae 1: 171 (1856); Miquel, Ned. Kruidk. Arch. 4: 113 (1856), '1859'; Bentham, Fl. Austral. 3: 446 (1867), p.p.; F. Mueller, Syst. Census Austral. Pl.: 76 (1882), Key Syst. Victorian Pl. 2: 30 (1885), Key Syst. Victorian Pl. 1: 292 (1888), Second Syst. Census Austral. Pl.: 128 (1889); Moore \& Betche, Handb. Fl. New South Wales: 253 (1893); Rodway, Tasmanian Fl.: 71 (1903); Anon., Field Naturalist Census Pl. Victoria: 60 (1931); Wakefield, Victorian Naturalist 72: 72 (1955); Black, Fl. South Australia, edn 2, 4: 800 (1957), p.p.; Beadle et al., Handb. Vasc. Pl. Sydney Distr.: 358 (1963), [see following Notes p.000]; Curtis, Stud. Fl. Tasmania 2: 273 (1963); Beadle et al., Fl. Sydney Region: 428 (1972).

Holotype: [Victoria], Port-Western, d'étroit de Bass, exped. de L'Astrolabe $1829 \mathrm{Mr}$ d'Urville (GDC); 'ad fretum Bass' (protologue).

SYNONYMY: Galium densum Hook. f., London J. Bot. 6: 461 bis (1847); Miquel, Ned. Kruidk. Arch. 4: 113 (1856), '1859'. LECTOTYPE (here designated): [Tasmania], Side of Western Mountains-Alt. 2000 ft., Gunn 549, 16.1.1845 (K). ISOLECTOTYPE: Gunn 549 (NSW). LECTOPARATYPES: [Tasmania], Hampshire Hills, Gunn 549, 1837 (K); on road to Macquarie Harbour from Lake St Clair, Gunn 549, 10.2.1845 (K).

Galium squalidum Hook. f., London J. Bot. 6: 462 bis (1847); J.D. Hooker, Fl. Tasmaniae 1: 171 (1856). Galium australe var. pilosohispidum Benth., Fl. Austral. 3: 447 (1867). LECTOTYPE (here designated): [Tasmania], New Norfolk, Gunn 1129, 6.11.1840 (K). IsOLECTOTYPE: Gunn II29 (NSW). LECTOPARATYPES: [Tasmania], Glen Leith, Gunn s.n., 14.9.1840 (K); Lawrenny, Gunn 1009, 10.1840 (K).

Galium albescens Hook, f., London J. Bot. 6: 462 bis (1847); J.D. Hooker, Fl. Tasmaniae 1: 171 (1856); Bentham, Fl. Austral. 3: 447 (1867); Curtis, Stud. Fl. Tasmania 2: 273 (1963). HolotyPE: Mount Wellington, Gunn s.n., 5.1839 (K). ISOTYPE: NSW.

[Galium australe var. laeve, nomen nudum: F. Muell. ex Hook. f., Fl. Tasmaniae 1: 171 (1856).

Galium subalatum, nomen nudum: F. Muell. ex Miq., Ned. Kruidk. Arch. 4: 113 (1856), '1859'.]

Misapplication of the name Galium australe: see misapplied names: 2. Galium terrae-reginae, 3. G. migrans and 10 . G. curvihirtum.

Distribution: In a variety of habitats at low altitudes, from coastal dunes to sheltered inland gullies (sometimes epiphytic on trunks of tree ferns) in Tasmania, South Australia (Kangaroo Island only), Victoria (E. of $146^{\circ} \mathrm{E}$ ) and southern coastal New South Wales. See fig. 1 .

Plant perennial, ? biennial; root system with a strong primary root and nodal roots up to $15 \mathrm{~cm}$ from rootstock; habit straggling or intertwining, sometimes loosely caespitose. Stems many to several, (5-) 20-60 (-100) $\mathrm{cm}$ long, branching in the lower half of plant; internodes (8-) 15-45 (-90) mm long; stem indumentum very variable, stems retrorsely aculeate, sparsely to densely covered with recurved or spreading hairs or rarely glabrous. Leaves elliptical to narrowly ovate or oblong, (4-) 6-12 (-18) mm long, 1.5-4 (-6) mm wide (stipular leaves slightly shorter), dark green to dark brown (on drying, usually coriaceous sometimes membranaceous); leaf-base angustate; leafapex acute to obtuse-mucronate; leaf-margin usually recurved sometimes revolute; upper surface with few to many forwardly directed hairs, occasionally with numerous soft spreading hairs or rarely almost glabrous; lower surface with spreading and/or forwardly directed hairs commonly restricted to the mid-vein, or sometimes glabrous; 


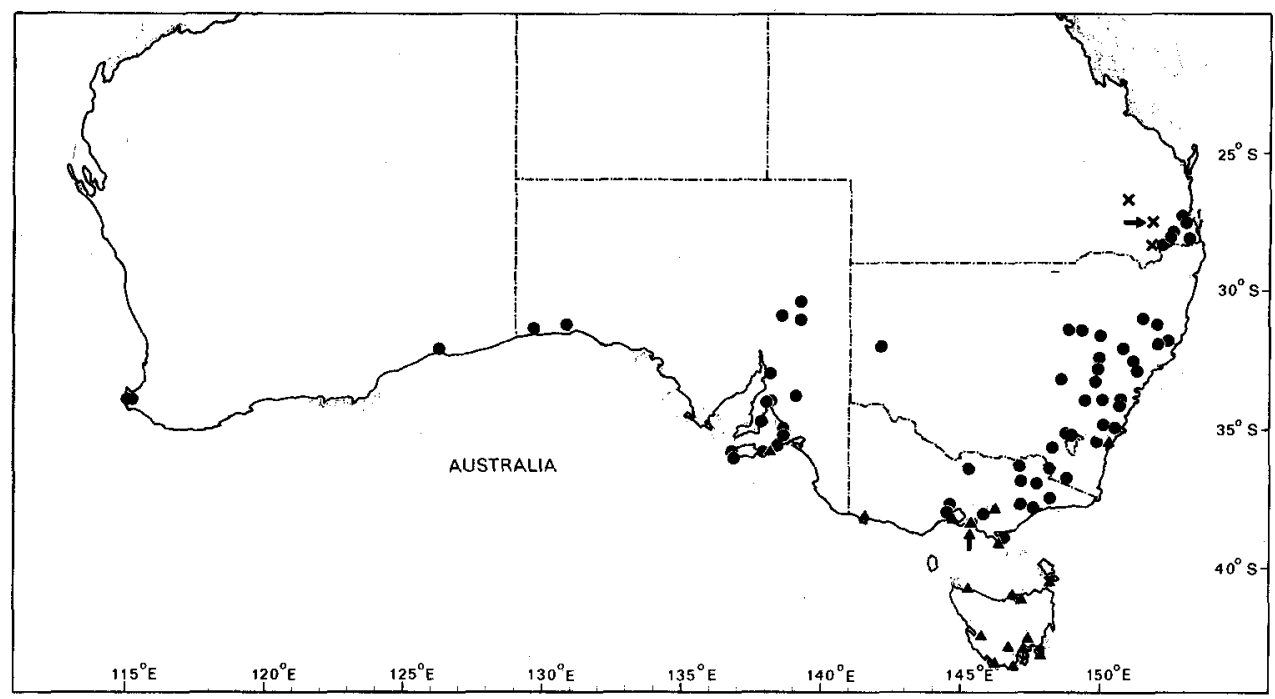

Fig. 1. Distribution of $G$, australe-A, G. terrae-reginae-X, and $G$. migrans- - . Arrows indicate type localities.

venation obscure except in membranaceous leaves; secretory cells usually coalescent and restricted to a subapical position, sometimes isolated and scattered, rarely absent. Inflorescence elongate, subcolumnar or pyramidal, (4-) 10-40 (-70) cm long, consisting of lateral and sometimes terminal cymes, vegetative growth continuing after the first flowering; lateral cymes dischasial or monochasial, (6-) 12-25 (-55) $\mathrm{mm}$ long; ultimate peduncles 1-7 (-10)-flowered, straight, sometimes bearing inconspicuous terminal bracts, (0-) 5-12 (-25) mm long; pedicels (0.5-) 2.5-10 (-22) mm long, slightly or strongly curved usually within $2 \mathrm{~mm}$ of ovary or fruit, rarely straight. Flowers (1.5-) 2.0-2.5 $(-2.8) \mathrm{mm}$ diameter, corolla rotate. Fruits $1.2-1.5 \mathrm{~mm}$ long, with numerous, usually transparent, uncinate hairs $0.4-1.0 \mathrm{~mm}$ long.

Selected Specimens: New South Wales-South Coast: Clyde district, Bäuerlen 1.1884 (MEL). VICTORIA: Fumina, Gippsland, 2.1925 (Ex Herb. Williamson, MEL); Queenscliff, Tadgell 10.1904 (MEL); "The Jackass", Fitzroy River, near Mount Deception, Beauglehole 1651, 1949 (NSW); ad ripam Darby fluminis promontorii Wilsoni, Mueller 3. 1853 (MEL); Near Darby River on Tongue Point Track, Wilsons Promotory, Johnson NSW 95298, 1.1967 (NSW). SouTH AUSTRALIA-Kangaroo Island: Ad rivulos rupestris sinus Pink-bay, Kangaroo Island, 3.1857 (MEL). TASMANIA: Clarke Island, Furneaux Group, Bass Strait, Maclaine, 1894 (MEL); “The Nut", Circular Head, far NW. Tasmania, Willis 1.1965 (MEL); Side of Bluff, Circular Head, Gunn 549/1842, 12.1837 (NSW); near George Town, Oakden 4.1886 (MEL); near Pipers River, SE. Coast (MEL); Gordon River, Milligan NSW 23919 (ex Herb. Archer), 12.1846 (NSW); Swanport, Story (MEL 17652, 17655, 17656); Rhyndaston, 12.1909 (MEL 17891, 17895); Junee Caves, near Maydena, T. \& J. Whaite 239I, 1.1961 (NSW); Mount Wellington, Gunn 549/1842, 1. 1841 (NSW); Port Arthur, Bufton, 1892 (MEL 17666, 17667, 17837); Cockle Creek, Recherche Bay, 1.1856 (MEL); New Harbour, Davis NSW 83665, 1.1938 (NSW); Gunner's Quoin, Rodway H 393, 6.1929 (CANB).

In the past the name Galium australe DC. has been applied broadly to include the Australian species having hairs on the ovaries or fruits; see 2. G. terrae-reginae, 3. G. migrans and 10. G. curvihirtum. Although it is included in Beadle et al. (1963), (1972), the species has not been authentically recorded from the Sydney district or the Blue Mountains.

The apically curved pedicels and the uncinate hairs of the fruits distinguish $G$. australe from other species.

\section{Galium terrae-reginae Ehrendorfer et McGillivray, sp. nov.}

Species affinis G. australis DC. sed habitu conferto, foliis brevibus, pilis fructuum curvatis non uncinatis differt.

Holotype: QueEnsland-Darling Downs District: One Tree Hill, Gowrie, F.M. Bailey (BRI 063832). 
Distribution: South eastern Queensland, from Chinchilla to Warwick. (No ecological data available). See fig. 1 .

Plant ? biennial to perennial; root system consisting of a strong primary root with nodal roots up to $5 \mathrm{~cm}$ from the rootstock; habit loosely caespitose to straggling. Stems numerous, $15-45 \mathrm{~cm}$ long, branching throughout, with numerous spreading hairs, internodes (4-) 8-20 (-25) mm long. Leaves ovate, narrowly ovate or elliptical, (2.5-) 3-5 (-7) mm long, (1.5-) 2-2.5 (-3.5) mm wide, dull green; leaf-base shortly attenuate; leaf-apex acute to shortly acuminate; leaf-margin recurved to strongly recurved; upper surface with evenly distributed spreading or ascending hairs, lower surface with slightly fewer hairs mainly on margin and mid-vein; reticulate venation usually evident; secretory cells inconspicuous and in a subterminal position only, or absent. Inflorescence subpyramidal to elongate; ultimate peduncles 1-3-flowered, 1-5 (-7) mm long, straight; pedicels $1-3(-5) \mathrm{mm}$ long, conspicuously curved near apex particularly at the fruiting stage. Flowers c. $2 \mathrm{~mm}$ diameter, corolla rotate, ? yellowish. Fruits c. $1.5 \mathrm{~mm}$ long, tuberculate to rugose and covered with numerous slightly to strongly curved hairs.

MISAPPLIED NAME: Galium australe auct. non DC: F.M. Bailey, Queensland Fl. 3: 782 (1900), p.p; F.M. Bailey, Compr. Cat. Queensland Pl.: 257 (1913).

SPecimens Examined: QueEnSland-Darling Downs District: Chinchilla, Darnell-Smith NSW 23986, 4. 1926 (NSW); Warwick, Beckler, 1857 (MEL); Prior's Station, Leichhardt NSW 23987, 11.1843 (NSW).

The species is characterized by its loosely caespitose to straggling habit, stems branching through their length, leaves usually not more than $5 \mathrm{~mm}$ long with inconspicuous secretory cells, cymes 1-3-flowered, pedicels conspicuously curved near the apex (especially evident at the fruiting stage) and fruits with numerous curved (not uncinate) hairs.

\section{Galium migrans Ehrendorfer et McGillivray, nom. nov.}

RePLACED SynONyM: Galium erythrorrhizum F. Muell. ex Miq., Ned. Kruidk. Arch. 4: 113 (1856), '1859', nomen illeg., non G. erythrorhizon Boiss. et Reut. 1852 ('erythrorrhizum' is an orthographic variant of the earlier epithet).

LECTOTYPE (here designated): [South Australia], Kangaroo Island, Mueller (U-sheet no. 2419B).

LECTOPARATYPE: [South Australia], in valle Schlanken, Behr (U).

SYNONYMY: (see also preceding replaced synonym): Galium gaudichaudii var. muriculatum Benth., Fl. Austral. 3: 446 (1867); F.M. Bailey, Queensland Fl. 3: 781 (1900); F.M. Bailey, Compr. Cat.: 257 (1913); Black, Fl. South Australia edn 2, 4: 799 (1957). Galium umbrosum var. muriculatum (Benth.) Ewart et Rees, Proc. Roy. Soc. Victoria (n.s.) 26: 4 (1913); Black, Fl. South Australia 4: 538 (1929); Ewart, Fl. Victoria: 1047 (1931). Galium umbrosum var. gaudichaudi-muriculatum Maiden et Betche, Census New South Wales Pl.: 188 (1916), nomen illeg. LECTOTYPE (here designated): New England, C. Stuart (K).

MisaPPlied NAMES: Galium australe auct. non DC.: Bentham, Fl. Austral. 3: 447 (1867), p.p.; Moore \& Betche, Handb. Fl. New South Wales: 253 (1893), p.p.; F.M. Bailey, Queensland Fl. 3: 782 (1900), p.p.; Black, Fl. South Australia 4: 539 (1929), p.p.; Gardner, Enum. Pl. Austral. Occid.: 122 (1931); Black, Fl. South Australia edn 2, 4: 800 (1957).

Galium umbrosum auct. non Forst. f. ex Hook. f. [nomen illeg. = G. propinquum]: F. Mueller, Fragm. 9: 188 (1875), p.p., Syst. Census Austral. Pl.: 142 (1882), p.p.; Key Syst. Victorian Pl. 2: 30 (1885), p.p., Key Syst. Victorian PI. 1: 292 (1888), p.p.; Maiden \& Betche, Census New South Wales Pl.: 188 (1916), p.p.; Black, Fl. South Australia 4: 538 (1929) p.p.; Ewart, Fl. Victoria: 1047 (1931), p.p.

Galium gaudichaudii auct. non DC.: Wakefield, Victorian Naturalist 72: 70 figs 4 \& 4a, 72 (1955), p.p.

DISTRIBUTION: Galium migrans is the most widely distributed species of Galium in Australia, occurring from south eastern Queensland through New South Wales, Victoria and South Australia to south western Western Australia; it is found up to altitudes of c. 1700 metres. Usually it grows in moist open or sheltered situations in rock crevices or over and between stones or boulders, frequently in association with limestone or basic igneous rocks. One may expect to find the species in the vicinity of limestone caves from New South Wales to south western Western Australia. See fig. 1.

Plants perennial, able to flower in the first year; root system with strong primary root and nodal roots up to $20 \mathrm{~cm}$ from the rootstock; habit straggling to loosely 
caespitose, sometimes pendulous in festoons. Stems numerous, (10-) 20-40 (-80) cm long, much-branched, branching sometimes reduced or absent in upper half of stems; internodes (5-) 15-40 (-100) $\mathrm{mm}$ long, stems commonly retrorsely aculeate, sometimes with many to few spreading to retrorse hairs, rarely glabrous. Leaves elliptical to ovate sometimes almost linear, (3-) 5-10 (-18) mm long, (1-) 1.5-3 (-5) mm wide, commonly a bright light green colour, sometimes darker and dull (from more shaded situations) or pale green with a greyish cast produced by the indumentum; leaf-base distinctly petiolate; leaf-apex obtuse-mucronate to shortly acuminate, sometimes acute; leaf margin recurved to almost flat; indumentum of few to many spreading or ascending hairs, evenly distributed on the upper surface, predominantly along margin and mid-vein on the lower surface; reticulate venation usually evident, more conspicuous on older leaves; secretory cells almost always present, commonly numerous and coalescent, particularly near the leaf-apex. Inflorescence pyramidal occupying about upper $2 / 3$ of stems; ultimate peduncles (2-) 3-7 (-25)-flowered, straight, (2-) 5-15 (-35) mm long; pedicels usually slightly curved, (1-) 2-3.5 (-8) mm long, peduncles and pedicels of preceding year's growth often persistent. Flowers 1.2-2.5 (-3) $\mathrm{mm}$ diameter, corolla rotate, cream to yellowish or white. Fruits $1.2-1.7 \mathrm{~mm}$ long exceedingly variable in surface covering: papillose, rugose, tuberculate or covered with curved to straight aculei or hairs.

Selected Specimens: Queensland-Moreton District: Mount Samson, c. 20 miles [32 km] NNW. of Brisbane, White 7.1913 (BRI); Three Mile Bush, c. 3 miles [5 km] NW. of Brisbane G.P.O., Bailey 7.1874 (BRI); Dams Creek, Mount Coot-tha ['Cootha'] near Brisbane, Everist 302, 1.1932 (BRI); Mount Tambourine, White 1.1916 (BRI); Mount Cordeaux, c. 25 miles [40 km] directly NE. by E. of Warwick, Phillips NSW 83632, 6.1961 (NSW); Christmas Creek, White 9320, 10.1933 (BRI); Roberts Plateau, Lamington National Park, Tryon \& White 2.1920 (BRI).-Darling Downs District: Spring Creek, near Killarney, Hubbard 5775, 3.1931 (BRI); The Ranch, foot of Wilsons Peak, Michael 11.1933 (BRI). NEW SouTH WALES-North Coast: Clarence River (MEL); Comboyne-Wingham, Phillips CBG 014082, 2.1961 (CBG); Bowman and Barrington Rivers, Maiden NSW 23904, 9.1897 (NSW); Brushy Mountains near Gloucester, Maiden NSW 23898, 9.1897 (NSW); Parsons Creek, S. of Bulga, Ehrendorfer 10.1966 (GRAZ, US); 0.5 miles [0.8 km] S. of Mount Bright Lookout, 4 miles [6 km] W. of Cessnock, McGillivray 1391, 9.1965 (NSW). -Central Coast: Nortons Basin, near Wallacia, Coveny NSW 83636, 10.1966 (NSW); Razorback Mountain, Camden, McBarron 9692, 10.1964 (NSW). -South Coast: Bungonia Gorge, S. of Marulan, Adams 1614, 1623, 11.1966 (CANB, NSW); Yalwal, c. 15 miles [24 km] by road WSW. of Nowra, Rodway 179, 11.1930 (NSW).-Northern Tablelands: Head of Macleay River, Leichhardt NSW 83625, 1843 (NSW); Moona Plains, Walcha, Crawford, 1.1885 (MEL); Tia Falls, Forsyth \& Cheel NSW 23906, 10.1900 (NSW); c. 8 miles [13 km] E. of Norfolk Falls, Warung State Forest, NE. of Coolah, Briggs 965 \& Johnson, 10.1966 (NSW); Warung State Forest, Ehrendorfer 10.1966 (GRAZ, WU).-Central Tablelands: Cheshire Creek, Wyagdon, Constable NSW 31184, 3.1955 (NSW); Jenolan Caves, Blakely NSW 23899 (NSW); 0.25 mile [0.4 km] W. of Abercrombie Caves, Briggs NSW 83634, 10.1965 (NSW). -Southern Tablelands: Wee Jasper Caves, Whaite 2834, 10.1964 (NSW); Two Sticks Road in vicinity of Devil's Gorge, A.C.T., Burbidge 1.1957 (CANB); near Mount Coree, A.C.T., Gray 4970, 1.1961 (CANB, NSW); Upper Orraral Valley, c. 1 mile [1.5 km] from homestead, A.C.T., Burbidge 6668, 11.1960 (CANB); Braidwood district, Bäuerlen 223, 12.1884 (MEL); Yarrangobilly Caves, Betche NSW 23982, 2.1897 (NSW); Near Geehi, c. 7 miles [11 km] NW. of Mount Kosciusko, Gittins 641, 1.1963 (NSW); Geehi River Track, below Pinnacle Track (c. W. of Mount Townsend), Ford NSW 83624, 1.1959 (NSW).-North Western Slopes: 1 mile [1.5 km] W. of Timor Rock, Warrumbungle Mountains, Johnson \& Briggs NSW 94574, 10.1966 (NSW); North side of Belougery Split Rock, Warrumbungle Mountains, Johnson \& Briggs NSW 94573, 10.1966 (NSW). - Central Western Slopes: Dart Brook, Scone, Cambage NSW 23911, 10.1909 (NSW); Wollar, near Mudgee, Campbell NSW 23897, 11.1906 (NSW); Apple Tree Flat, Cudgegong, c. 10 miles [16 km] SE. of Mudgee, Tindale NSW 25656, 10.1953 (NSW); Bingham Springs, Bumberry, Cleland 9.1916 (AD). - South Far Western Plains: Scropes Range, Menindee district, Constable NSW 4878, 11.1947 (NSW). Victoria: Snowy Creek, Ovens River, Morton, 1889 (MEL); Junction Dam, Kiewa River, Rodd 369, 12.1966 (NSW); Snowy River, Bäuerlen 158, 4.1890 (MEL 17848, top specimen only); Limestone Creek; E. of Benambra, Willis 2.1946 (MEL); Murrindal River, Buchan district, Wakefield NSW 83629, 10.1949 (NSW); Gippsland, Nightingale Creek, just above Tali Karng, Muir 2947, 12.1963 (MEL); Bairnsdale district, Mitchell River Cliffs, near Wy Yung, Hart 11.1917 (MEL); Trentham Falls, Robbins (Beauglehole 7735), 1938 (NSW); Wilsons Promontory, Mueller NSW 17853, 5.1853 (NSW); Warragul Forest, Reader 11.1885 (MEL); Bacchus Marsh, Meebold 21896, 12.1936 (NSW); Lederberg, Gorge, Sutton 10.1904 (MEL); You Yangs, Willis 11.1962 (MEL). SouTH AuSTRALIA-Nullarbor Region: Murrawijinie Caves, 6 miles [10 km] $\mathrm{N}$. of Nullarbor homestead, Willis 8.1947 (MEL); Koonalda Cave, 55 miles [88 km] ENE. of Eucla, Beauglehole 13419, 9.1965 (NSW).-Flinders Ranges Region: Gorge of western branch of Balcanoona Creek above Loch Ness Well, Gammon Ranges, Eichler 12920, 9.1956 (AD); Mount Chambers Gorge, c. $95 \mathrm{~km}$ SE. of Leigh Creek, Filson 3463, 10.1960 (AD); Oratunga Creek, near Moolooloo, c. $20 \mathrm{~km}$ NE. of Parachilna, Lothian 948, 10.1960 (AD); Wirrabara Forest, Gill 12 (MEL).-Eyre Peninsula Region: c. $2 \mathrm{~km} \mathrm{~N}$. of Tumby Bay, Lothian 1145, 10.1962-Northern Lofty Region: Port Germein Gorge, c. 10 km E. of Port Germein, Cleland 9.1963 (AD); South Hummocks Range, c. $110 \mathrm{~km} \mathrm{NNW.} \mathrm{of} \mathrm{Adelaide,}$ 
Kraehenbuehl 1402, 8.1965 (AD).-Murray Region: Worlds End, SE. of Burra, Brummitt 9.1893 (AD).Yorke Peninsula: c. $5 \mathrm{~km}$ W. of Kulpara, S. side of Kadina Road, northern Yorke Peninsula, Lothian 3045, 11.1964 (AD); Yorke Peninsula, Tepper 729, 1133 (MEL).-Southern Lofty Region: Morialta, c. $10 \mathrm{~km}$ E. of Adelaide, Black 9.1928 (AD); Third Creek, Lofty Ranges, Mueller 1.1848 (MEL); Waterfall Gully, base of Mounty Lofty, Tate 10.1878 (AD); National Park, Belair, c. $8 \mathrm{~km}$ SSE. of Adelaide, Black 11.1905 (AD): Between Cape Jervis and Victor Harbour, south-eastern slope to Boat Harbour Creek, c. $31 / 2 \mathrm{~km} \mathrm{~N}$. of the sea coast, Eichler 14443, 11.1957 (AD).-Kangaroo Island: Cape Willoughby, Tepper 1278 (MEL); Ravine des Casoars, $8 \mathrm{~km}$ E. of Cape Borda, Wilson 683, 11.1958 (AD); Near small lagoon SW. of Kelly Hill reserve, c. $12 \mathrm{~km}$ ENE. of Cape du Couedic, Wilson 708, 11.1958 (AD). WESTERN AuSTRAlIA-Darling District: Lakes Cave, Margaret River, Ehrendorfer 12.1966 (NSW, WU); Mammoth Cave, Margaret River, Ehrendorfer 12.1966 (NSW, WU); Gallingup, Maiden NSW 84540, 10.1909 (NSW).-Coolgardie District: 9 miles [14 km] S. of Cocklebiddy, George 8550, 10.1966 (NSW).

Galium migrans is not only the most widely distributed of the Australian species of Galium but also the most variable, particularly in the nature of the indumentum of its fruits. It is characterized by a well-developed primary root, much-branched habit, light bright green foliage (except in well-shaded situations), leaves obtusemucronate to acuminate, inflorescences with conspicuous ultimate peduncles and gently curved pedicels, the persistence of old peduncles and pedicels on older stems.

4. Galium propinquum A. Cunn., Ann. Nat. Hist. 2: 205 (1839); J.D. Hooker, Fl. Novae-Zelandiae 1: 113 (1852); Wakefield, Victorian Naturalist 72: 70 fig. 3 \& 3a, 71 (1955); Allan, Fl. New Zealand 1: 592 (1961); Beadle et al., Handb. Vasc. Pl. Sydney Distr.: 257 (1963), Fl. Sydney Region: 427 (1972).

Holotype: New Zealand: No $469 R$. Cunningham 1834. (K). 'Shaded woods, Wangaroa, R. Cunningham, 1833' (protologue).

SYNONYMY: Galium umbrosum Forst. f. ex Hook. f., Handb. New Zealand Fl. 1: 121 (1864), nomen illeg.; [G. Forster, Prodr.: 89 (1786), nomen nudum,; A. Cunningham, Ann. Nat. Hist. 2: 206 (1839), nomen nudum, pro syn.; Endlicher, Ann. Wiener Mus. 1: 177 (1836), nomen nudum; J.D. Hooker, Fl. Novae-Zelandiae 1: 113 (1852), nomen nudum, pro syn.]; F. Mueller, Fragm. 9: 188 (1875), sens. ampl., p.p., Syst. Census Austral. Pl.: 76 (1882), p.p., Key Syst. Victorian PI. 2: 30 (1885), p.p., Key Syst. Victorian Pl. 1: 292 (1888), p.p., Second Syst. Census: 128 (1889), p.p.; Kirk, Student's Fl. New Zealand: 249 (1899); Rodway, Tasmanian Fl.: 71 (1903), sens. ampl., p.p.; Cheeseman, Man. New Zealand Fl.: 266 (1906); Maiden \& Betche, Census New South Wales Pl: 188 (1916); Cheeseman, Man. New Zealand Fl., edn 2: 879 (1925); Black, Fl. South Australia 4: 538 (1929), sens. ampl., p.p.; Ewart, Fl. Victoria: 1047 (1931), sens. ampl., p.p.; Black, Fl. South Australia, edn 2, 4: 799 (1957). TYPE: as for Galium propinquum A. Cunn.

Galium propinquum var. elongatum Hook. f., Fl. Novae-Zelandiae 1: 113 (1852), ' $\alpha$. 'elongata'.

Galium propinquum var. glabratum Hook. f., l.c. ' $\beta$. glabrata'.

Galium propinquum var. hispidulum Hook. f., l.c., ' $\gamma$. hispidula'; Allan, Fl. New Zealand 1: 592 (1961). Hooker did not at any time cite types for the three preceding varietal names.

Galium gaudichaudii var. muriculatum Benth., Fl. Austral. 3: 446 (1867), p.p.; Galium umbrosum var. muriculatum (Benth.) Ewart et Rees, Proc. Roy. Soc. Victoria (n.s.) 26: 4 (1913); Black, Fl. South Australia 4: 538 (1929), p.p.; Ewart, Fl. Victoria, 1047 (1931), p.p. LeCTOTYPE: see synonymy of 3. G. migrans. Galium erythrocaulon Colenso, Trans. New Zealand Inst. 16: 332 (1884); Allan, Fl. New Zealand 1: 592 (1961). TYPE: NEW ZEALAND: 'Stony declivities, skirts of dry woods between Norsewood and Danneverke, Waipawa county, 1879-1882', Colenso. (WELT, not seen). IsOTYPE (?): K.

DiSTRIBUTION: New Zealand: North and South Islands, Stewart Island and Ruapuke Island-occurring in a variety of habitats but favouring grassy or rocky sites in shady and/or moist situations. See fig 2 .

Australia: Queensland (rarely collected), New South Wales, Victoria and South Australia (only one collection, from the Mount Lofty Ranges). In coastal climates it favours moist shaded fertile sites in rainforest or wet sclerophyll forest, while on the tablelands it occurs also in more open situations in woodland and grassland. The species has not been recorded in association with limestone. See fig. 2 .

Plants annual (?), to perennial; root system of primary root later supplemented or superseded by nodal roots from well-developed slender rhizomes; habit prostrate to weakly decumbent. Stems usually weak and flexible, (4-) 6-30 (-50) cm long, sparingly branched; internodes (3-) 10-30 (-45) mm long; stems commonly retrorsely aculeate to glabrous or with spreading to retrorse hairs. Leaves elliptical to narrowly ovate, (1.5-) 2-8 (-15) mm long, (0.7-) 1-2.5 (-6) mm wide, dull green, to yellowish green, rarely dark green; leaf-base tapering to a short petiole; leaf-apex shortly acuminate to obtuse-mucronate, sometimes acute; leaf margin recurved to strongly recurved; upper surface with few spreading to ascending hairs, lower surface glabrous or with few hairs along margin and mid-vein; venation usually obscure on upper surface, commonly visible on lower surface with reflected light, usually evident with 


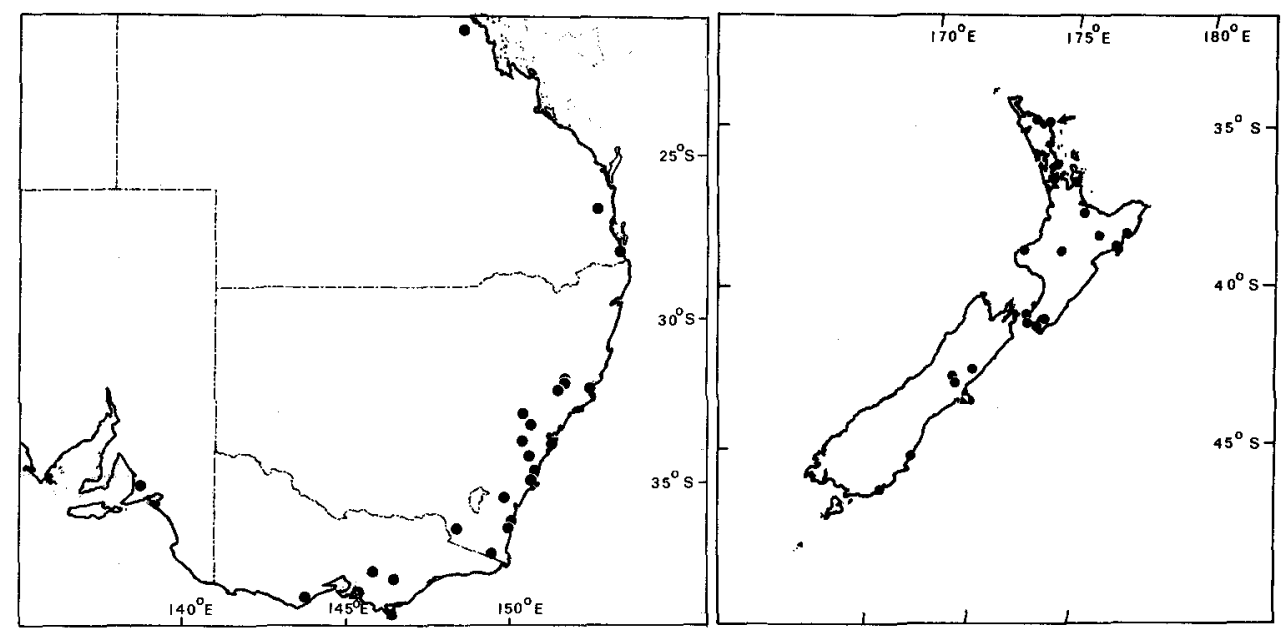

Fig. 2. Distribution of G. propinquum. Arrow indicates the type locality.

transmitted daylight; secretory cells always present, usually linear, occasionally roundish, coalescent towards the leaf apex and along the mid-vein, frequently also scattered over the lower surface. Inflorescence elongate sometimes pyramidal, consisting of axillary cymes; ultimate peduncles 1-5 (-7)-flowered, commonly 3-flowered, often absent or inconspicuous, usually straight, maximum length $6 \mathrm{~mm}$; pedicels (0.3-) 1-4 (-7) mm long, often curved. Flowers (1.0-) 1.2-1.8 (-2.0) mm diameter, corolla rotate, white to cream. Fruits $0.9-1.2 \mathrm{~mm}$ long, papillose, tuberculate (with pointed tubercles), ruminate, rugose (with thin-walled rugosities), or covered with few to many curved ascending hairs, sometimes exhibiting a combination of these surface features.

Selected Specimens: New Zealand (N. to S.; all specimens are from, CHR unless otherwise indicated):-North Island: Cape Brett, Bay of Islands, Collett 9.1964; Whangeripo Valley, Petrie 11.1919; Kawau Island, Moore 12.1953; Auckland, Kirk 42 (MEL); Pongakawa Valley, Te Puke district, Healy 11.1948; Anaura Bay, Zotov 2.1940; Mount Maungapohata, Druce 12.1953; Near Mahnia camp-site Tongariro National Park, Ogle 2.1962; Morere Hot Springs Reserve, Mason 8672, 1.1961; Egmont, Druce 2.1960; East Wairarapa, Pahaoa Road, Moore 11.1958; Mount Maunsell, East Wairarapa, Druce, 11.1962; Rimutaka Hill, Hay 11.1951; Colonial Knob, Tawa Flat, Allan 11.1943; Otari Bush, Wellington, Mason 12.1939; Miramar Gully, Wellington, Mason 8.1947; Tongue Point, Wellington Heads, Healy 4.1937; Putangirua Stm., Palliser Bay, Hay 3.1952; Cape Turakirae, Wellington, Moore 12.1952.-South Island: Middle Gorge, Hurunui River, North Canterbury, Moar 856, 8.1957; Lees Valley, behind Mount Oxford, Simpson 4.1956; View Hill, Oxford, Mitchell 12.1942; Godley Head, Banks Peninsula, Macmillan 65/13 \& Collett 1.1965; Presqu'ile de Banks, Raoul, 1843 (MEL); Moeraki Point, East Otago, Collett 3.1965; Tautuku Beach, Catlins district, Mason 4550, 1.1957; Cunningham 115 (MEL).

Australia: QueEnsland-South Kennedy District: Dalrymple Heights, Clemens, Jul-Nov. 1947 (BRI).-Wide Bay District: Blackall Range, Keys 5.1910 (BRI), White, 12.1916 (NSW). - Moreton District: Tamborine Mountain, c. 45 miles $[70 \mathrm{~km}] \mathrm{S}$. of Brisbane, Hubbard 5.1930 (BRI). New SouTH WALES -North Coast: Copeland, near Gloucester, Hind NSW 84348, 1.1967 (NSW); Gloucester River, below Gloucester Tops, WSW. of Gloucester, Constable 6000, 6.1965 (NSW); Nabiac via Taree, Gilbert NSW 23902, 7.1947 (NSW).-Central Coast: Culoul Range, 8 miles [13 km] W. of Putty Road, Rodd 365, 12.1966 (NSW); Manly Beach, Wilhelmi 12.1863 (MEL); Fox Valley, Wahroonga, Johnson 945, 4.1947 (NSW); The Oaks Recreation Reserve, McBarron 13847, 2.1967 (NSW); Macquarie Pass, Albion Park, McBarron 10283, 1.1965 (NSW); Minnamurra Falls, Jamberoo, McBarron 5320, 11.1950 (NSW); Saddelback Mt., Jamberoo-Kiama Rd, McBarron 10275. 1.1965 (NSW).-South Coast: Nowra, Rodway 2123, 2.1936 (NSW); Nariri Creek, Reader, 1880 (MEL); Bodalla State Forest, 5 miles [ 8 km] NW. of Narooma, Constable 6785, 5.1966 (NSW); Mt Dromedary, Reader (MEL); Nungatta, Weatherhead, 1883 (MEL).-Northern Tablelands: Barrington Tops, Fraser \& Vickery NSW 23905, 5.1934 (NSW). - Central Tablelands: E. of Rylstone, McGillivray 1088 \& Rodd, 4.1965 (NSW); base of Scenic Railway, Katoomba, Briggs 882 \& Johnson, 10.1966 (NSW).--Southern Tablelands: Braidwood district, Bäuerlen 179, 12.1886 (MEL); head of Spencers Creek, Kosciusko, Johnson \& Constable NSW 15942, 1.1957 (NSW). VICTORIA: Acheron Way, Tadgell, 3.1937 (MEL); East Gippsland, Wakefield 4323, 1945-1946 (NSW); Watts River, Mueller 12.1873 (MEL); Thompson River crossing S. of Walhalla, Johnson NSW 95323, 1.1967 (NSW); Forrest, Otway Ranges, Beauglehole 7742, 2.1952 (NSW); c. 1 mile $[1.5 \mathrm{~km}]$ E. of Lilly Pilly Gully, Wilsons Promontory, Tindale NSW 95251, 1.1967 (NSW); Tidal River, Wilsons Promontory, Johnson NSW 95297, 1.1967 (NSW). South Australia: Mount Lofty Ranges, Menzel 11.1897 (AD). 
The species is characterized by its prostrate to weakly decumbent habit, welldeveloped rhizomes frequently with the primary roots not evident, weak sparinglybranched stems, commonly 2-5-flowered pedunculate to shortly pedunculate cymes, and curved pedicels. It may be distinguished from Galium migrans by its welldeveloped rhizomes and weak, sparingly-branched comparatively glabrous stems; from Galium ciliare by its prostrate to weakly decumbent stems, leaves tapering to a short distinct petiole, ultimate peduncles 1-5 (-7)-flowered, and fruits which are usually not smooth. Galium trilobum, the endemic New Zealand species, has stems usually longer than $20 \mathrm{~cm}$, leaves rarely less than $6 \mathrm{~mm}$ long and without secretory cells, and curved peduncles longer than $5 \mathrm{~mm}$.

5. Galium ciliare Hook. f., London J. Bot. 6: 461 bis (1847); J.D. Hooker, Fl. Tasmaniae 1: 170, t. XLI A, B (1856); Miquel, Ned. Kruidk. Arch. 4: 113 (1856), '1859'; J.D. Hooker, Handb. New Zealand Fl. 1: 121 (1864); Bentham, Fl. Austral. 3: 446 (1867); F. Mueller, Fragm. 9: 188 (1875), pro syn.; Curtis, Stud. Fl. Tasmania 2: 272 (1963); Burbidge \& Gray, Fl. Austral. Cap. Territory: 339 (1970).

TYPE MATERIAL: Kew Negative No. 9184, (K). 'Dry pastures, abundant; Gunn.: - v.v. .' (protologue). [Selection of a lectotype for the name Galium ciliare cannot be justified and would only need to be considered if infraspecific names were proposed. In the original description Hooker cites his own and Gunn's collections but without any details which can be matched with herbarium material. The citations in Flora Tasmaniae do not clarify this. In addition, the specimens and the collection data are so arranged on the type sheet, Kew Negative No. 9184, that one cannot be assigned to the other with any confidence. Certainly a piece or pieces could be designated as a lectotype, but in the present circumstances this seems both unnecessary and inadvisable. Bentham, l.c. p. 446, in citing 'abundant in dry pastures, J.D. Hooker', is not lectotypifying the name but merely mentioning Hooker's field note and indicating that he has seen material from Hooker's collections, or , in this example, material from Herbarium Hookerianum including Gunn's collections.]

Synonymy: Galium gaudichaudii var. glabrescens Benth., Fl. Austral. 3: 446 (1867); F.M. Bailey, Compr. Cat. Queensland Pl.: 257 (1913); Domin, Biblioth. Bot. 89: 1184 (1929). Galium umbrosum var. gaudichaudi-glabrescens Maiden et Betche, Census New South Wales Pl.: 188 (1916), nomen illeg. HolotyPE: New South Wales, Clarence River, Beckler (K). 'New England, Beckler' (protologue). IsOTYPE: (MEL 17762).

Galium umbrosum Forst. f. ex Hook. f., Handb. New Zealand Fl. 1: 121 (1864), nomen illeg., sens. ampl.: F. Mueller, Fragm. 9: 188 (1875), p.p., Syst. Census: 76 (1882), p.p., Key Syst. Victorian Pl. 2: 30 (1885), p.p., Key Syst. Victorian Pl. 1: 292 (1888), p.p., Second Syst. Census: 128 (1889), p.p.; Moore \& Betche, Handb. Fl. New South Wales: 253 (1893); Rodway, Tasmanian Fl.: 71 (1903), p.p.

Galium gaudichaudii var. latifolia, nomen nudum: Miq., Ned. Kruidk. Arch. 4 : 113 (1856), '1859'.

Misapplication of the name Galium ciliare: See misapplied names: 7. Galium compactum. (Some material of Galium ciliare has been identified as $G$. gaudichaudii, but in the literature it is treated as G. gaudichaudii var. glabrescens).

DISTRIBUTION: In savannah and woodland habitats intermingled with grasses or sometimes between rocks, favouring basaltic soils in tableland regions; altitudinal range c. 100-1300 metres. Bunya Mountains, Queensland; tablelands (rarely slopes) in New South Wales, montane situations in Victoria, and Tasmania. See fig. 3.

Plants perennial; root system of nodal roots from subterranean stems, primary root not conspicuous; habit spreading, trailing, sometimes loosely caespitose. Stems ascending or erect from trailing primary stems, unbranched or sparingly branched near base, (4-) 10-20 (-40) cm long; internodes (6-) 8-18 (-25) mm long, stems with spreading dense to sparse hairs or glabrous, not retrorsely aculeate. Leaves ovate, sometimes rotund, usually maintaining a light yellowish-green colour on drying, (2.5-) 3.5-8 (-10) mm long, (1-) 1.5-3 (-4) mm wide, abruptly narrowed into an obtuse base; apex obtuse-mucronate to obtuse; margin recurved; upper surface noticeably glossy, with spreading or forwardly directed hairs along margin and midrib, or glabrous; reticulate venation usually evident; secretory cells, several to many, linear and scattered as well as coalescent near the apex. Inflorescence elongate, columnar, (5-) 8-20 (-25) $\mathrm{mm}$ long, consisting of lateral and terminal cymes, the latter sometimes prominent; ultimate peduncles 5-9-flowered, 5-15 mm long, straight; pedicels straight, 1-5 mm long. Flowers commonly yellowish, occasionally cream (? sometimes white), 1.5-3 $\mathrm{mm}$ diameter, corolla rotate. Fruits papillose but otherwise smooth.

Selected Specimens: QueEnSland-Darling Downs District: Summit of Mount Mowbullen, Bunya Mountains, Tryon 11.1890 (BRI). NEw SouTH WALES-Northern Tablelands: 19 miles [30 km] NE. of 


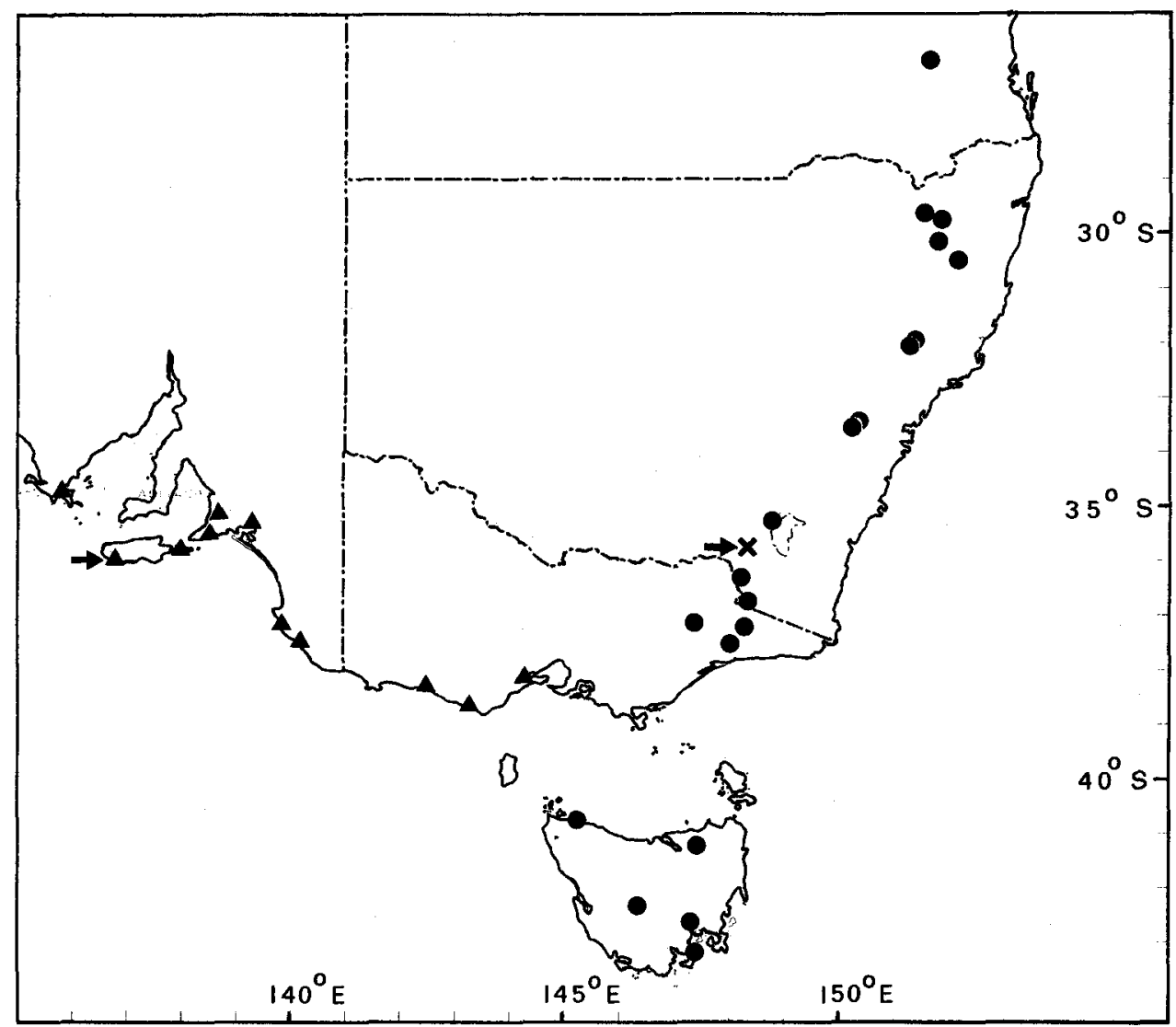

Fig. 3. Distribution of $G$. ciliare- , G. compactum-A, and G. roddii-X. Arrows indicate type localities.

Glen Innes on Gwydir Highway, McGillivray 2393, 9.1966 (NSW); Glen Innes, Betche NSW 23913, 10.1886 (NSW); Ben Lomond, Beckler (MEL); Ebor Falls, 50 miles $[80 \mathrm{~km}]$ NE. of Armidale, Davis (ex Herb. Rodway) NSW 83980, 1.1941 (NSW). -Central Tablelands: Mt Tomah, Rodd NSW 94577, 11.1966 (NSW); Hartley Vale Railway Station, Johnson \& Briggs 889, 891, 10.1966 (NSW). - Southern Tablelands: Dingo Dell area, N. of Mount Coree, A.C.T., Burbidge 6869, 12.1960 (CANB); Alpine Way just S. of Youngal Saddle in Snowy Mountains, Muir 325I, 2.1964 (MEL); Ingeegoodbee, Costin NSW 23925, 2.1949 (NSW).-Central Western Slopes: Wooloma Mountain, Belltrees, Scone, White NSW 23912, 11.1903 (NSW); Moonan Brook, Hunter River, Carter 1.1895 (MEL). VICTORIA: Gelantipy, c. 25 miles [40 km] directly W. of Bonang, Wakefield NSW 83652, 1.1948 (NSW); W. Tree, c. 13 miles [21 km] N. of Buchan, Wakefield NSW 83651, 1.1949 (NSW); Dargo Track, Mount St. Bernard, Tadgell 12.1914 (MEL). Tasmania: Bluff, Circular Head, (ex Herb. Gunn) 12.1837 (NSW); Mount Arthur, Coates \& Sullivan, 1886 (MEL); Mount Barrow and foothills, Dorset County, Rupp NSW 23927, 1.1922 (NSW); Mersey River, Story (MEL); 1 mile [1.5 km] W. of Dee River, Lyell Highway, Johnson NSW 23926, 1.1949 (NSW); Rhyndaston, 12.1909 (MEL); North Bruni Island, Rodway NSW 83985, 1.1901 (NSW); Marlborough at Glen Burn,Gunn 1128, 1.1847 (NSW); Pinnibar area, Mazanec 3.1963 (CANB).

Galium ciliare is characterized by relatively erect and unbranched stems, glossy ovate to rotund leaves, columnar inflorescences often with prominent terminal cymes and yellow to cream flowers.

6. Galium gaudichaudii $D C$., Prodr. 4: 607 (1830); Bentham, Fl. Austral. 3: 446 (1867); F.M. Bailey, Queensland Fl. 3: 781 (1900); Rodway, Tasmanian Fl.: 71 (1903); F.M. Bailey, Compr. Cat. Queensland Pl.: 257 (1913); Ewart \& Rees, Proc. Roy. Soc. Victoria 26: 4 (1913); Anon., Field Naturalists Census Pl. Victoria: 60 (1923), p.p.; Black Fl. South Australia 4: 538 (1929); Domin, Biblioth. Bot. 89: 1189 (1929); Ewart, Fl. Victoria: 1046 (1931); Wakefield, Victorian Naturalist 72: 72 (1955); Black, 


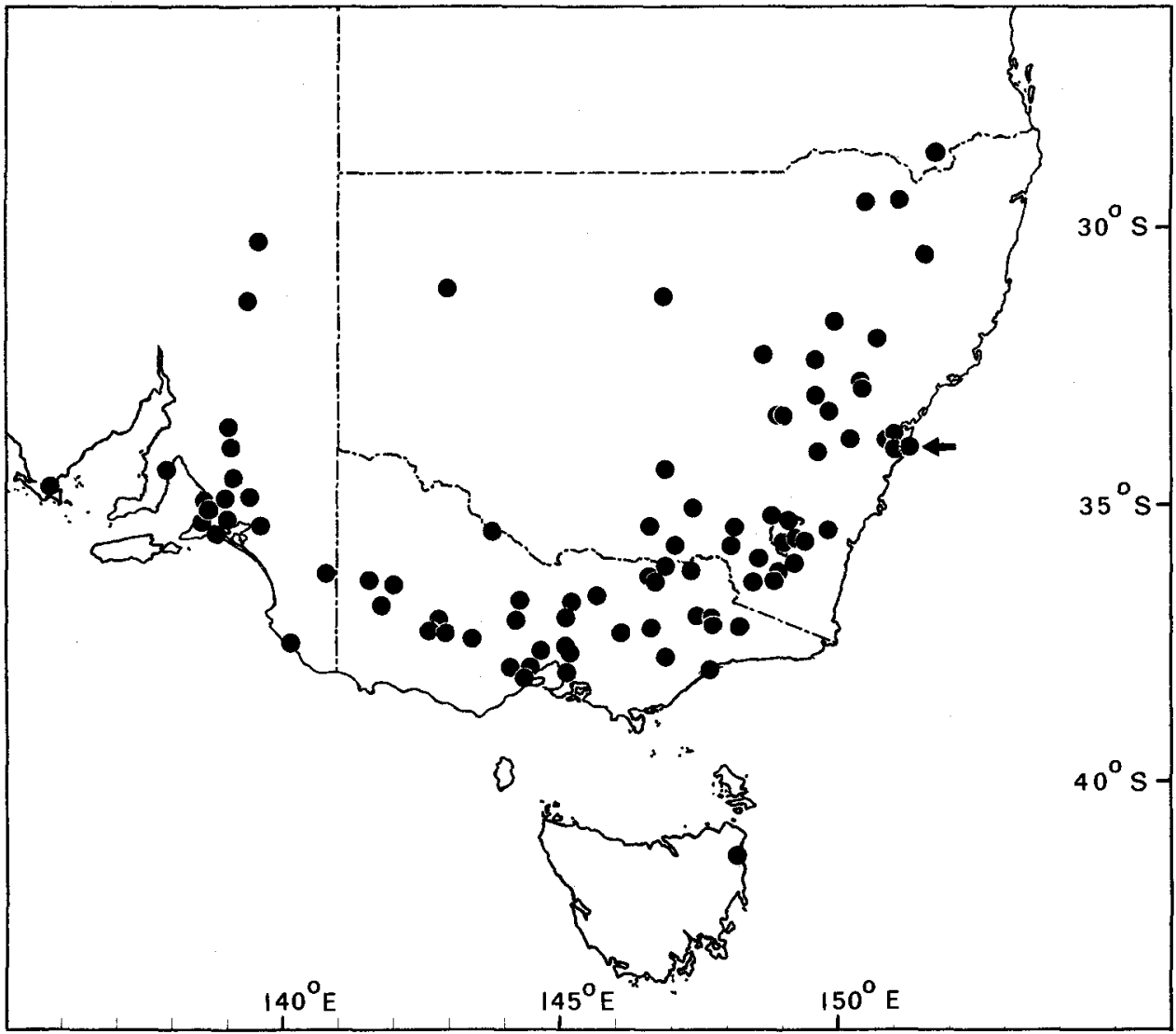

Fig. 4. Distribution of G. gaudichaudii. Arrow indicates the type locality.

Fl. South Australia, edn 2, 4: 799 (1957), p.p.; Beadle et al., Handb. Vasc. Pl. Sydney Distr.: 358 (1963); Cochrane et al., Fl. \& Pl. Victoria: 78, plate 215 (1968); Burbidge \& Gray, Fl. Austral. Cap. Terr.: 339, fig. 343 (1970); Beadle et al., Fl. Sydney Region: 428 (1972).

HOLOTYPE: [New South Wales], in Nova-Hollandia ad Port Jackson, Gaudichaud (G-DC). IsOTYPES (?): E, K (ex Herb. J. Gay).

SyNONYMY: Galium vagans Hook. f., London J. Bot. 6: 461 bis (1847). HolotyPE: Van Diemen's Land, Gunn 548 (K) ['Grassy places; Gunn' (protologue).]

Galium axiflorum F. Muell. ex Miq., Nederl. Kruidk. Arch. 4: 113 (1856), '1859', including:

forma minor Miq. HoLOTYPE: [South Australia], Lofty-range, Mueller (U).

forma laxe-ramosum Miq. HoLOTYPE: [South Australia], Barossa-range, in formatione arenacei transitionis, Mueller (U).

forma procerum Miq. HOLOTYPE: Tasmania, Stuart (U).

Galium umbrosum var. gaudichaudii (DC.) Maiden et Betche, Census New South Wales PI.: 188 (1916), 'Gaudichaudi'. TYPE: as for G. gaudichaudii DC.

Galium gaudichaudii var. typicum Domin, Biblioth. Bot. 89: 1184 (1929), nomen inval.

MiSAPPLIED NAME: Galium umbrosum auct. non Forst. f. ex Hook. f., nomen illeg. [superfl., $=G$. propinquum]: F. Mueller, Fragm. 9: 188 (1875), p.p., Syst. Census: 76 (1882), p.p.; C. Moore, Census Pl. New South Wales: 32 (1884), in parenthesis; F. Mueller, Key Syst. Victorian Pl. 2: 30 (1885), p.p., Key Syst. Victorian PI. 1: 292 (1888), p.p., Second Syst. Census: 128 (1889), p.p.; Moore \& Betche, Handb. Fl. New South Wales: 253 (1893), p.p.; Rodway, Tasmanian Fl.: 71 (1903), p.p.; Maiden \& Betche, Census New South Wales Pl.: 188 (1916), p.p.

Misapplication of the name Galium gaudichaudii: see misapplied names: 3. G. migrans and 7. G. compactum. 
Distribution: A widely distributed species in south eastern Australia, usually occurring on relatively dry sites in sclerophyll forest, woodland, savannah or heath and on a variety of soil types from those based on acid to basic igneous rocks to those of sedimentary origin, including limestones, sandstones, shales and slates. See fig. 4.

Plants perennial; root system with strong primary root and weak development of nodal roots up to $5 \mathrm{~cm}$ from the rootstock; habit caespitose, loosely caespitose, straggling to trailing. Stems numerous, commonly erect, (5-) $10-25(-100) \mathrm{cm}$ long, branching in the lower half of plant; internodes (5-) 12-20 (-35) mm long; stems hirsute, retrorsely aculeate, or sometimes almost glabrous. Leaves narrowly ovate to linear (often almost narrowly triangular in outline owing to recurving of margins towards apex), often irregularly curved in drying, (4-) 5-8 (-12) mm long, (0.6-) 0.8-1.2 (-2.0) $\mathrm{mm}$ wide; leaf-base shortly attenuate to truncate, non-petiolate; leaf-apex subobtuse (but when observed superficially appearing acute owing to recurving of the leaf margin); leaf-margin strongly recurved; upper surface with scattered ascending hairs, hairs on lower surface confined to margin and midvein; venation obscure; secretory cells coalescent in a subapical position, sometimes also isolated and scattered. Inflorescence elongate, columnar or subpyramidal; lateral cymes condensed, 3-5 (-10) mm long; ultimate peduncles 1-5 (-7)-flowered, up to $2.5 \mathrm{~mm}$ long; pedicels $0.1-1.0$ $(-2.0) \mathrm{mm}$ long. Flowers $1.5-2 \mathrm{~mm}$ diameter, corolla rotate, cream, purplish in bud. Fruits $0.8-1.5 \mathrm{~mm}$ long, rugulose, without tubercles or hairs.

Selected Specimens: Queensland-Darling Downs District: Granite Hill just W. of Glen Aplin, Everist \& Webb 1371, 11.1946 (BRI, CANB). NEW SOUTH WALES-Central Coast: near Vineyard Station, Richmond Line, Salasoo 990, 9.1952 (NSW); Veterinary Research Station, Glenfield, McBarron 8407, 10.1963 (NSW); Bringelly, Boorman \& Cheel NSW 23957, 9.1913 (NSW).-Couth Coast: Narira Creek, Reader 8.1880 (MEL).- Northern Tablelands: 5 miles [8 km] NW. of Armidale, Davis (ex Herb. Rodway) NSW 38600, 12.1940 (NSW); c. 8 miles [13 km] E. of Norfolk Falls, Warung State Forest, NE. of Coolah, Johnson \& Briggs 964, 10.1966 (NSW).-Central Tablelands: Hill End, Lauterer 9.1885 (MEL); Mount Canobolas, 10 miles [16 km] SW. of Orange, Constable NSW 53344, 11.1960 (NSW); Limekilns, c. 17 miles [27 km] by road NE. of Bathurst, Whaite 878, 1.1951 (NSW); Jenolan Caves, Blakely (SYD); Rockley, Boorman NSW 23959, 11.1906 (NSW); Abercrombie Caves, Boorman NSW 23958, 11.1918 (NSW).Southern Tablelands: 17 miles [27 km] from Uriarra on Yass Road, Moore 1849, 10.1952 (CANB); Hall, A.C.T. D’Arnay 49, 11.1959 (CANB); Canberra-Broken Bridge (Uriarra), A.C.T., Melvaine 10.1938 (CANB); Canberra, [A.C.T.] Cambage 2984, 11.1911 (NSW, SYD); Black Mountain, A.C.T., Gauba CBG 010972, 11.1949 (CBG); Red Hill (Canberra), A.C.T., Gauba 10.1949 (GAUBA 9565; CBG 010934); slopes of Jerrabomberra, S. of Queanbeyan, Burbidge 6699, 11.1960 (CANB); top of Fitz's Hill, A.C.T., eastern side near summit, Burbidge 6217 \& Gray, 12.1958 (CANB); 2 miles [3 km] from Naas on Gudgenby Road, A.C.T., Moore 2969, 11.1954 (CANB); Tharwa, [A.C.T.], Cambage 3003, 11.1911 (NSW); Braidwood district, Bäuerlen 12.1884, 1.1885, $10.1886(\mathrm{MEL}) ; 2$ miles [3 km] S. of Captains Flat, Briggs NSW 83602, 12.1965 (NSW); The Glen, via Tumbarumba, McBarron 2884, 12.1948 (NSW, SYD); Happy Jacks River, Tumut River catchment area, Newman NSW 23915, 12.1952 (NSW); Snowy Mountains Highway near Rhine Falls, 12 miles [19 km] from Cooma, Muir 2422, 10.1961 (MEL); Murrumbidgee River, 5 miles [8 km] from Cooma, Cambage 2107, 11.1908 (NSW, SYD); Jindabyne, Maiden \& Forsyth NSW 23971, 1.1899 (NSW); Summit Road, Sawpit Creek, Mt Kosciusko area, Martin 1.1963 (SYD).North Western Slopes: Warialda, Rupp NSW 23976, 9.1905 (NSW); Inverell Road and Frasers Creek, Boorman NSW 23978, 9.1910 (NSW).-Central Western Slopes: Kars Gap, Scone, Cambage 2600, 10.1909 (SYD); Dubbo, Cambage NSW 23954, 10.1906 (NSW); Camboon, 7 miles [11 km] N. of Rylstone, Baker NSW 23956; 10.1893 (NSW); Bowan Park near Cudal, Blakely NSW 23967, 10.1906 (NSW); Ardlethan, Cambage 4332, 10.1916 (NSW). - Southern Western Slopes: Gilmore, near Tumut, Boorman NSW 23941, 10.1916 (NSW); Pleasant Hills, Fisher, 1890 (MEL); Woomargama, McBarron 131, 12.1946 (SYD); Wagga Wagga, Thom, 1887 (MEL); Monument Hill, Albury, McBarron 3748, 10.1949.-North Western Plains: Girilambone, Betche 84, 10.1886 (NSW). - North Far Western Plains: Tarella, [a station N. of Wilcannia], Bäuerlen 8.1887 (MEL). VICTORIA: McKellops Bridge, Robbins (Beauglehole 7734), c. 1937 (NSW); Boundary Creek, below the "Bare Rock", Wulgulmerang, far East Gippsland, Willis 11.1962 (MEL); on the Omeo-Corryong road, 8 miles $[13 \mathrm{~km}] \mathrm{S}$. of Carmody's Hut, Muir 3665, 12.1964 (MEL); between Omeo and Swifts Creek, Robbins (Beauglehole 7736), c. 1937 (NSW); Buckety Plain, Bogong High Plains, Willis 1.1947 (MEL); along Langstone Creek on metamorphic granite soils up to $4000 \mathrm{ft}$. [1200 m], Stirling 10.1882 (MEL 17705); 4 miles [6 km] S. of Granya, 23 miles [37 km] E. of Wodonga, Muir 2371, 10.1961 (MEL); Beechworth, Meebold 21705, 11.1936 (NSW); Mount Stanley, McBarron 2930, 1.1949 (NSW); Minogue's Lookout, 10 miles [16 km] SE. of Mount Howitt, Muir 994, 12.1959 (MEL); Tali Karng, Gippsland, Muir 2966, 12.1963 (MEL); Sperm Whale Head, Gippsland, Barton 11.1926(MEL); near Still Creek, on the Jamieson Road, 22 miles [35 km] from Eildon, Muir 1640, 10.1960 (MEL); Hurstbridge, Tadgell 10.1923 (MEL); Warrandyte, Meebold 21704, 11.1936 (NSW); Cheltenham, Morrison 11.1894 (AD, CANB); Darebin Creek, Mueller 10.1852 (MEL); Tamleugh, Goulburn Valley, Minchin, 1886 (MEL); Hughes Creek, near Avenal, Davis NSW 83606 (ex Herb. Rodway) 11.1942 (NSW); Seymour, Davis NSW 83607 (ex Herb. Rodway) 9.1942 (NSW); Lower Loddon (near junction with Murray), Thom, 1882 (MEL); Flora Hill, Bendigo, Robbins (Beauglehole 7980), 10.1947 (NSW); Castlemaine, Robbins (Beauglehole 
3715), 10.1939 (NSW); Taradale, Tadgell 10.1933 (MEL); Toolern Vale, Tadgell 12.1932 (MEL); Lintons Forest, Whan NSW 23974, 1862 (NSW); Stawell, Parch 10.1928 (MEL); Ararat, Green 54 (MEL); along Forest Access Road to Ararat near top of William Ridge, Grampians area, Whaite 1618, 11.1953 (NSW); Moyston, Sullivan 10.1872, 10.1879 (MEL); Ben Major State Forest, N. of Beaufort, Muir 1589, 10.1960 (MEL); Salt Lake, Shire of Dimboola, Reader, 11.1895 (MEL 17722, in part); Mount Arapiles, Beauglehole 7579, 10.1964 (NSW); Mitre Rock, near Mount Arapiles, c. 15 km WSW. of Horsham, Hicks 9.1960 (AD); Nhill, Staer NSW 23945, 4.1911 (NSW); Maryvale, Macpherson, 1898 (MEL); Lower Glenelg River, between Eaglehawk Bend and Blackfish Creek, Willis 10.1948 (MEL). South Australia-Eyre Peninsula Region: Port Lincoln, Browne, 1874 (MEL), Black 10.1909 (AD).-Flinders Ranges Region: Ridge N. of North Tusk, Gammon Ranges, Eichler 12813, 9.1956 (AD); Wilpena Pound, Beck 10.1924 (AD).Northern Lofty Region: Mine Hill, Burra, Brummitt 10.1892 (AD); Tothill Range, c. $145 \mathrm{~km} \mathrm{~N}$. of Adelaide, Kraehenbuhl 1151, 10.1963 (AD).-Murray Region: Mannum, Murray River, Cleland NSW 23966, 11.1913 (NSW).- Yorke Peninsula: Ardrossan, Tepper (ex Herb. Black) 10.1879 (AD). - Southern Lofty Region: Barossa Range, Behr (MEL); Port Adelaide, Blandowsky, 1850 (MEL); Belair, Tate 11.1883 (AD); National Park, Belair, Eichler 13393, 12.1956 (AD); Mount Lofty Botanic Garden, c. $12 \mathrm{~km}$ SE. of Adelaide, Kuchel 1260, 10.1963 (AD); Strathalbyn, Black 11.1910 (AD); Willunga, Black 9.1904 (AD); Encounter Bay, Cleland (ex Herb. Black) 1.1924 (AD). TASMANIA: Mount Direction, Black 11.1911 (MEL); St. Helens, Fitzgerald NSW 23937; South Esk, Stuart, 1848 (MEL); Landfall Creek, Simson 10.1880 (MEL).

Galium gaudichaudii may be distinguished from other species by its strong primary root with little or no development of nodal roots, leaves commonly disarrayed in drying and narrowly ovate to narrowly triangular, with margins strongly recurved towards the leaf apices, relatively unbranched stems bearing crowded cymes with peduncles usually less than $2.5 \mathrm{~mm}$ long and pedicels up to $2 \mathrm{~mm}$ long; the fruits are rugulose, without hairs or tubercles. Specimens of several species, principally those of 3. G. migrans and 7. G. compactum, have been misclassified as G. gaudichaudii.

\section{Galium compactum Ehrendorfer et McGillivray, sp. nov.}

Species affinis G. gaudichaudii DC. sed rhizomatibus conspicuis, ramorum internodiis foliisque brevibus, cymis paucifloris 1-2 (-3) floribus differt.

Holotype: SOutH Australla: Kangaroo Island, Sandhill on road to Cape du Couedic, ca. $11 \frac{1}{2} \mathrm{~km}$ south of Rocky River Homestead, Hj. Eichler 15376, 11.11.1958 (AD).

Distribution: Coastal, commonly sandy habitats, from near Geelong in Victoria to Port Lincoln, South Australia, and including Kangaroo Island. See fig. 3.

Plants perennial; root system of strong primary root and well-developed rhizomes; habit caespitose, sometimes straggling, ? sometimes pendulous. Stems usually numerous (2-) 3-8 $(-25) \mathrm{cm}$ long, pilose to almost glabrous, not retrorsely aculeate; internodes (2-) 3-8 (-15) mm long, generally shorter than in the other species. Leaves ovate to narrowly ovate, (2.5-) 3.5-4.5 (-6) $\mathrm{mm}$ long and (0.8-) 1.5-1.8 (-2.2) mm wide, light green or brown, never dark on drying; leaf-base cuneate to attenuate; leaf-apex acute; margin recurved sometimes more prominently so towards the leaf-apex; upper surface pilose to almost glabrous, not retrorsely aculeate; indumentum on lower surface similar but occurring predominantly on the margin and midvein; venation obscure; secretory cells usually present and coalescent near the leaf-apex, sometimes in scattered or continuous groups. Inflorescence columnar, sometimes catkin-like, (0.5-) 1-4 (-8) cm long, consisting of lateral and terminal cymes; lateral cymes shorter than the leaves; ultimate peduncles up to $0.5 \mathrm{~mm}$ long, 1-2 (-3)-flowered; pedicels up to c. $0.5 \mathrm{~mm}$ Flowers $1.5-2 \mathrm{~mm}$ diameter, corolla rotate. Fruits c. $1.5 \mathrm{~mm}$ long, rugose.

MisaPPLIED NAMES: Galium ciliare auct. non Hook. f.: Black, FI. South Australia 4: 538 (1929). It differs noticeably from $G$. ciliare which has a looser habit (internodes usually $8-18 \mathrm{~mm}$ long), longer lateral cymes (peduncles 5-15 mm long), and papillose fruits.

Galium gaudichaudii auct. non DC.: Black, Fl. South Australia edn 2, 4: 799 (1957), p.p.; G. gaudichaudii has little or no development of rhizomes, looser habit (internodes usually $12-20 \mathrm{~mm}$ long), and longer cymes 3-5 (-10) mm long.

Selected SPeCimens: Victoria: Near Geelong, Wilson, 1885 (MEL); Gellibrand River, Anon. 1874 (MEL); Warrnambool, Anon. 10.1856 (NSW); Cape Nelson, Anon. 15 (MEL). SouTH AUSTRALIA-Eyre Peninsula: Port Lincoln, Dixon 10.1883 (AD), Black 10.1909 (AD).-Murray Region: Scrub E. of Wellington, Tepper (AD). - Southern Lofty Region: Belair, Tate 11.1883 (AD); Waterfall Gully, c. 10 km ESE. of Adelaide, Cleland 11.1924 (AD); Summerton near Uraidla, Griffith 12.1907 (AD); Willunga, Black, 9.1904 (AD); Cape Jervis, Maiden NSW 23935, 1.1907 (NSW); Newland Head, Encounter Bay, 
Cleland 1.1928 (AD)._Kangaroo Island: Dudley Peninsula, Tepper 11.1883 (AD); Rocky River, Cleland 12.1934 (AD); Cape du Couedic, Cleland 11.1924 (AD); Mount Taylor, Tepper 11.1886 (MEL).-Southeastern Region: Robe, Beck 10.1910 (AD); Beachport, Black 11.1917, 12.1917 (AD).

This species is characterized by well-developed rhizomes, compact habit (most stems $3-8 \mathrm{~cm}$ long), columnar inflorescences with lateral cymes shorter than the leaves, and rugose fruits.

\section{Galium roddii Ehrendorfer et McGillivray, sp. nov.}

Species affinis G. gaudichaudii DC. sed rhizomatibus conspicuis, fructibus tuberculatis differt.

HolOTYPE: NEw SOUth WALES: Cave Creek, Cooleman Caves, east of Yarrangobilly, c. 0.5 mile $[0.8$ $\mathrm{km}$ ] below the Blue Waterhole, Rodd 87, 18.4.1965. (NSW). IsOTYPES (yet to be distributed): E, K, US, WU.

DiSTRIBUTION: The species is a narrow endemic, apparently confined to limestone in the vicinity of Cave Creek, Cooleman Caves east of Yarrangobilly in the Southern Tablelands of New South Wales at the comparatively high altitude of $1200-1250$ metres. It occurs as occasional clumps in crevices of cliffs and limestone pavements, and in gravel at the foot of clefts in the limestone. See fig. 3.

Plants perennial; root system mainly roots from well-developed rhizomes, primary root strongly developed but rarely observed; habit caespitose with some trailing stems, and clumps at intervals along the rhizomes. Stems several to many, 6-30 cm long, hirsute, much-branched near the base, sparingly branched or unbranched in the upper two-thirds; internodes (2-) 4-12 (-25) mm long. Leaves narrowly ovate, curved upwards, 3-4.5 mm long, 0.8-1.2 mm wide, dull pale green, soon becoming brown on lower stems; leaf base narrowing abruptly to a broad short petiole; leaf apex acute, accentuated by the recurved leaf margin; margin thickened, recurved; upper surface with few to many spreading or ascending hairs, lower surface lineate, usually with fewer hairs; venation obscure; secretory cells linear to round, coalescent and commonly also diffuse, rarely absent. Inflorescence columnar, consisting of many short lateral cymes 2-3 mm long; ultimate peduncles 1-2 (-3)-flowered, 0.3-0.5 mm long; pedicels 0.3-0.5 mm long. Flowers c. $2.2 \mathrm{~mm}$ diameter, corolla rotate, dull cream. Fruits 1.3-1.6 $\mathrm{mm}$ long, covered with numerous rounded pale-capped tubercles.

SPECIMENS EXAMined: New South Wales--Southern Tablelands: Cave Creek, about 2 miles [3 km] above junction with Goodradigbee R., Rodd 476, 3.1967 (NSW); Cave Ck., head of Goodradigbee R., Rodd 563, 12.1967 (NSW).

The species is named after A.N. (Tony) Rodd, Botanist, whose collections and critical notes have improved our understanding of many taxa in the Australian flora.

9. Galium binifolium Wakefield, Victorian Naturalist 72: 69, 70 fig. 1 (1955); Beadle et al., Handb. Vasc. Pl. Sydney Distr.: 357 (1963), Fl. Sydney Region: 427 (1972).

HolOTYPE: VICTORIA: Sand-dunes near Betka River, Mallacoota, beneath a stand of tall Melaleuca shrubs, Wakefield 4794, 6.1.1953 (MEL). IsOTYPE: $\mathrm{K}$.'

SynONymy: ? Galium umbrosum var. bifolium F. Muell., Fragm. 9: 188 (1875). TyPE: Hastings-River, Beckler. Not seen.

MisAPPlied NAMEs: ? Galium geminifolium auct. non. F. Muell.: Domin, Biblioth. Bot. 89: 1184 (1929).

DisTRIBUTION: On slopes and near creeks in forest and woodland entangled in herbage or trailing over rocks, occurring from near sea-level up to c. 800 metres altitude. Eastern New South Wales, Victoria and south eastern South Australia. See fig. 5.

Plants biennial or annual (? perennial); root system of strong to rather weak primary roots sometimes supplemented by occasional nodal roots; habit straggling to clambering, sometimes loosely caespitose. Stems several to occasionally many, (15-) $20-50(-100) \mathrm{cm}$ long, sparingly branched or unbranched; internodes (20-) 30-60 (-80) $\mathrm{mm}$ long, stem surface with retrorsely aculeate asperities or smooth, apparently never with a conspicuous indumentum. Leaves often reclinate to retrorse, narrowly elliptical, commonly slightly falcate, (4-) 6-12 (-20) mm long, (0.7-) 1-2.5 (-3) mm wide, stipular leaves rarely exceeding two-thirds the length of non-stipular leaves; texture 


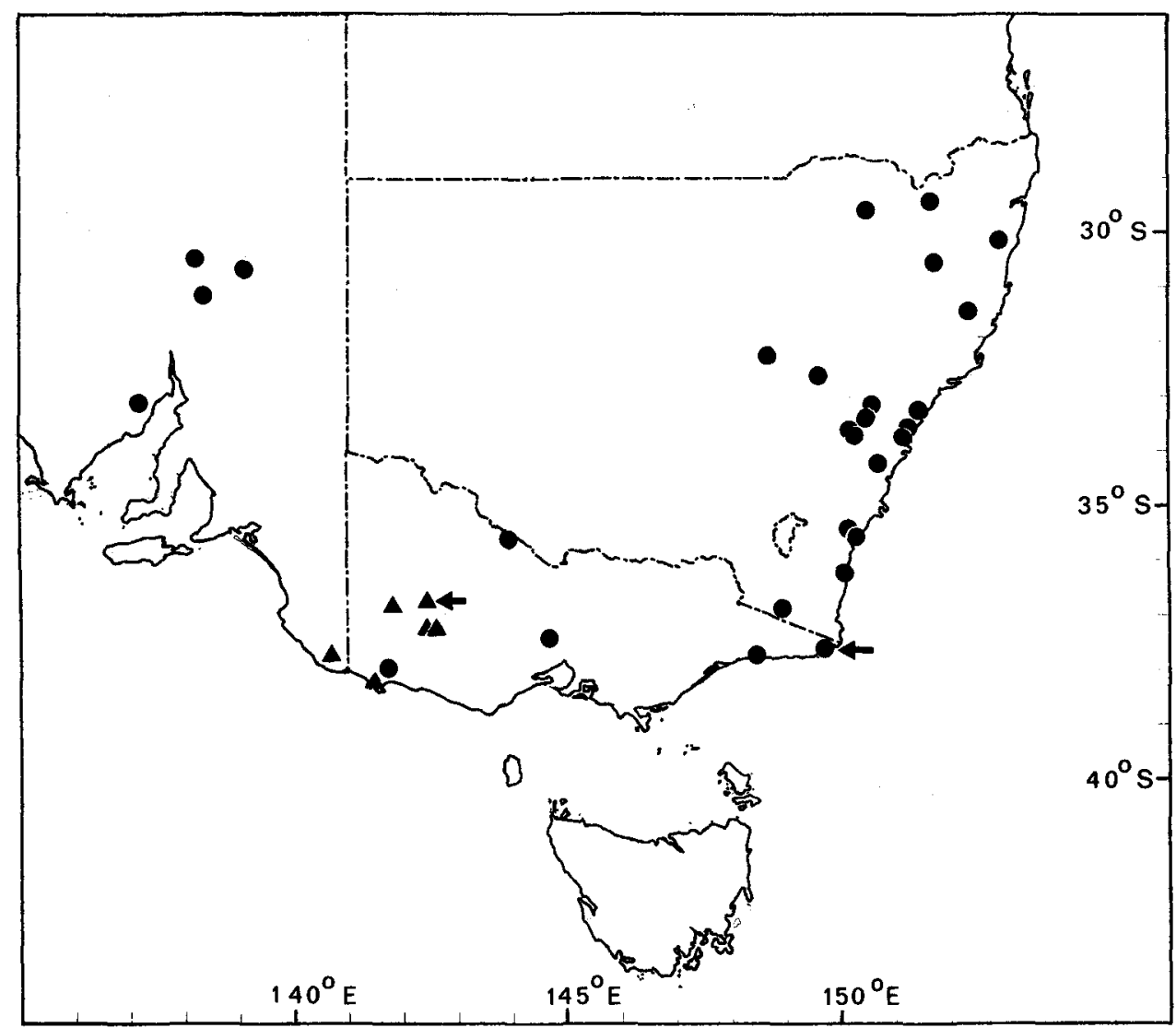

Fig. 5. Distribution of $G$. binifolium- , and $G$. curvihirtum-A. Arrows indicate type localities.

membranaceous to subcoriaceous; leaf-base attenuate to shortly attenuate; leaf-apex acute; margins recurved; leaf-surface with few forwardly directed hairs mainly on margins and on the upper and lower surfaces of the midrib; reticulate venation usually clearly visable; some apical secretory cells usually present. Inflorescence elongate from well below the middle of stems (above the middle in some young plants); lateral cymes c. 5-25 mm long; ultimate peduncles conspicuous, straight to slightly curved, commonly 3-flowered, but up to c. 8-flowered, pedicels up to $5 \mathrm{~mm}$ long, commonly 2-3 mm. Flowers 1.8-2.2 (-2.5) $\mathrm{mm}$ diameter, corolla rotate, cream to yellowish sometimes with reddish or purple tinges. Fruits c. $1.5 \mathrm{~mm}$ long, rugose or sometimes ribbed, glabrous or rarely with numerous curved short hairs.

MisAPPLIED NAMES: Galium umbrosum var. muriculatum auct. non (Benth.) Ewart et Rees: Black, Fl. South Australia 4: 538 (1929), p.p.

Galium gaudichaudii var. muriculatum auct. non Benth.: Black, Fl. South Australia, edn 2, 4: 799 (1957), p.p.

Selected SPecimens: New South Wales--North Coast: Timber Top via Glenreagh, King NSW 23988, 11.1949 (NSW); Macleay River, Beckler (MEL); Seaview Range, Maiden NSW 23910, 11.1897 (NSW); Upper Williams River, Fraser \& Vickery NSW 23903, 1.1934 (NSW)._Central Coast: Culoul Range, 9 miles [14 km] W. of Putty road, Rodd 367, 12.1966 (NSW); Wyong-Warnervale, Salasoo 1619, 10.1958 (NSW); Milson Island, Hawkesbury River, Cleland (AD); Mountain Lagoon, 10 miles [16 km] NE. of Bilpin, Constable 5764, 4.1965 (NSW); Warrawee, Blakely NSW 23900, 11.1914 (NSW); Nepean River, Douglas Park, McBarron 11827, 1.1966 (NSW).--South Coast: Clyde River, Bäuerlen 103, 11.1884 (MEL); Kioloa State Forest, NE. of Batemans Bay, Adams 1590, 10.1966 (NSW); Narira Creek, near Narooma, Reader 8.1880 (MEL); Tingiringi Mountain, Bäuerlen 170, 1.1889 (MEL)-Northern Tablelands: 5 miles [8 km] NE. of Armidale, Davis NSW 83986, 5.1939 (NSW)._Central Tablelands: Mount Victoria, Maiden NSW 23901, 12.1896 (NSW); base of Scenic Railway, Katoomba, Johnson \& Briggs 886, 10.1966 (NSW).- 
North Western Slopes: Emmaville, Boorman NSW 23989, 10.1901 (NSW); Warialda, Boorman NSW 83658, 10.1914 (NSW);-Central Western Slopes: Dubbo, Cleland 9.1911 (AD); Moolarben Creek, Mudgee-Wollar road, Johnson \& Constable NSW 31908, 9.1950 (NSW). VICTORIA: Lake Charm, French Jnr. 11.1896 (MEL); Hanging Rock, Hesket, Tindale NSW 84368, 1.1967 (NSW); Mount Cole, Dillon 31, 1894 (MEL); Wall Creek, Orbost, Wakefield 3954, c. 10.1945 (NSW); near Lake Condah, Beauglehole 7744, 11.1950 (NSW). SouTh AUSTRAlia-Flinders Ranges Region: Mt Parry c. $15 \mathrm{~km} \mathrm{W.} \mathrm{of} \mathrm{Leigh} \mathrm{Creek,} \mathrm{Tate,} \mathrm{8.1883}$ (AD); c. 8 km E. of Nepabunna Station, Lothian 3220, 11.1964 (AD); Parachilna Gorge, Black 10.1918 (AD).-Locality uncertain: Middle Back, Rockwater holes, Tepper 9.1886 (AD).

This species is characterized by its sparingly branched, smooth to retrorsely aculeate stems, the binifoliate condition of its leaves [i.e. the foliaceous stipules noticeably shorter than the corresponding leaves of each whorl], conspicuous peduncles and relatively short pedicels, and its rugose fruits.

It has been confused with the binifoliate Asperula aesthenes Shaw et Turrill ( $A$. geminifolia var. retroscabra F. Muell, Fragm. 9: 188 (1875), nomen nudum), sometimes misidentified as $A$. geminifolia $\mathrm{F}$. Muell. In $A$. aesthenes the angles of the stems have a keen scabrous feel and the flowers are tubular; it is readily distinguished from the smoother-stemmed $G$. binifolium.

The occasional occurrence of fruits with hair suggests that the fruits surface of the Australian species should not be considered to have overriding significance in delimiting taxa. In $G$. binifolium the hairy and glabrous fruits can occur on intertwining plants in the same piece of herbage (cf. Fraser \& Vickery NSW 23903).

\section{Galium curvihirtum Ehrendorfer et McGillivray, sp. nov.}

Species affinis $G$. binifolii Wakefield sed folii margine revoluto venis obscuris, pilis fructuum numerosis curvatis differt.

Holotype: Victoria: Mt. Arapiles, ca. 20 miles [32 km] east of Horsham, Aston 1075, 3.10.1963 (MEL 17791).

Distribution: From the brief ecological notes available this species grows in open forest or woodland as an inconspicuous herb amongst herbaceous ground vegetation. It occurs in western Victoria and the south east of South Australia. See fig. 5 .

Plants biennial, ? perennial; root system with well-developed primary root, nodal roots absent; habit prostrate to straggling, rarely caespitose. Stems several, rarely numerous, (10-) 25-40 (-110) cm long, commonly retrorsely aculeate, sometimes with spreading or retrorse hairs or glabrous; internodes (10-) 15-35 (-60) $\mathrm{mm}$ long. Leaves usually narrowly ovate-linear, sometimes ovate or elliptical, (3-) 4-12 (-15) mm long, (0.6-) 1-2 (-2.5) mm wide; leaf-base attenuate; leaf-apex narrowly acute sometimes acuminate; leaf-margin strongly recurved sometimes revolute; upper surface with few, sometimes many, spreading to ascending hairs; lower surface usually glabrous except for sparse indumentum along the midvein; midvein conspicuous, reticulum obscure, rarely evident; secretory cells usually linear, both coalescent in a subapical position and scattered, sometimes also confluent along the midvein. Inflorescence elongate to loosely pyramidal, occupying the upper half of the plant, consisting of lateral, rarely terminal cymes; ultimate peduncles (1-) 2-3 (-5)-flowered, straight, (0.5-) 1-6 $(-11) \mathrm{mm}$ long, pedicels straight or slightly curved $0.5-1.5(-2.5) \mathrm{mm}$ long. Flowers white to cream c. $2 \mathrm{~mm}$ diameter, corolla rotate. Fruits (1.0-) $1.5 \mathrm{~mm}$ long, densely covered with curled whitish hairs (finer and shorter than those of Galium australe).

MiSAPPLIED NAME: Galium australe auct. non DC.: Black, Fl. South Australia 4: 539 (1929); edn 2, 4: 800 (1957).

SPECIMENS EXAMINED: VICTORIA: Mount Arapiles, Reader 11.1895 (MEL); Mount Arapiles, Beauglehole 7437,7439 \& Finck, 2.1959 (NSW); Mount William, Grampians, Sullivan 11.1872 (MEL); East side of Victoria Range, gully above Strachans, Grampians, Beauglehole 16119 \& Willis, 12.1966 (NSW); Gorae West, W. of Portland, Beauglehole 1652, 10.1946 (NSW); Bridgewater, W. of Portland, Beauglehole 1654, 10.1946 (NSW). South AUSTRALIA-Southeastern Region: Monbulla Scrub, near Mount Gambier, Andrew 12.1918 (AD).

This species is characterized by its sparse, straggling, sparingly-branched habit, 1-3 $(-5)$-flowered cymes and fruits covered with numerous curled whitish hairs. 


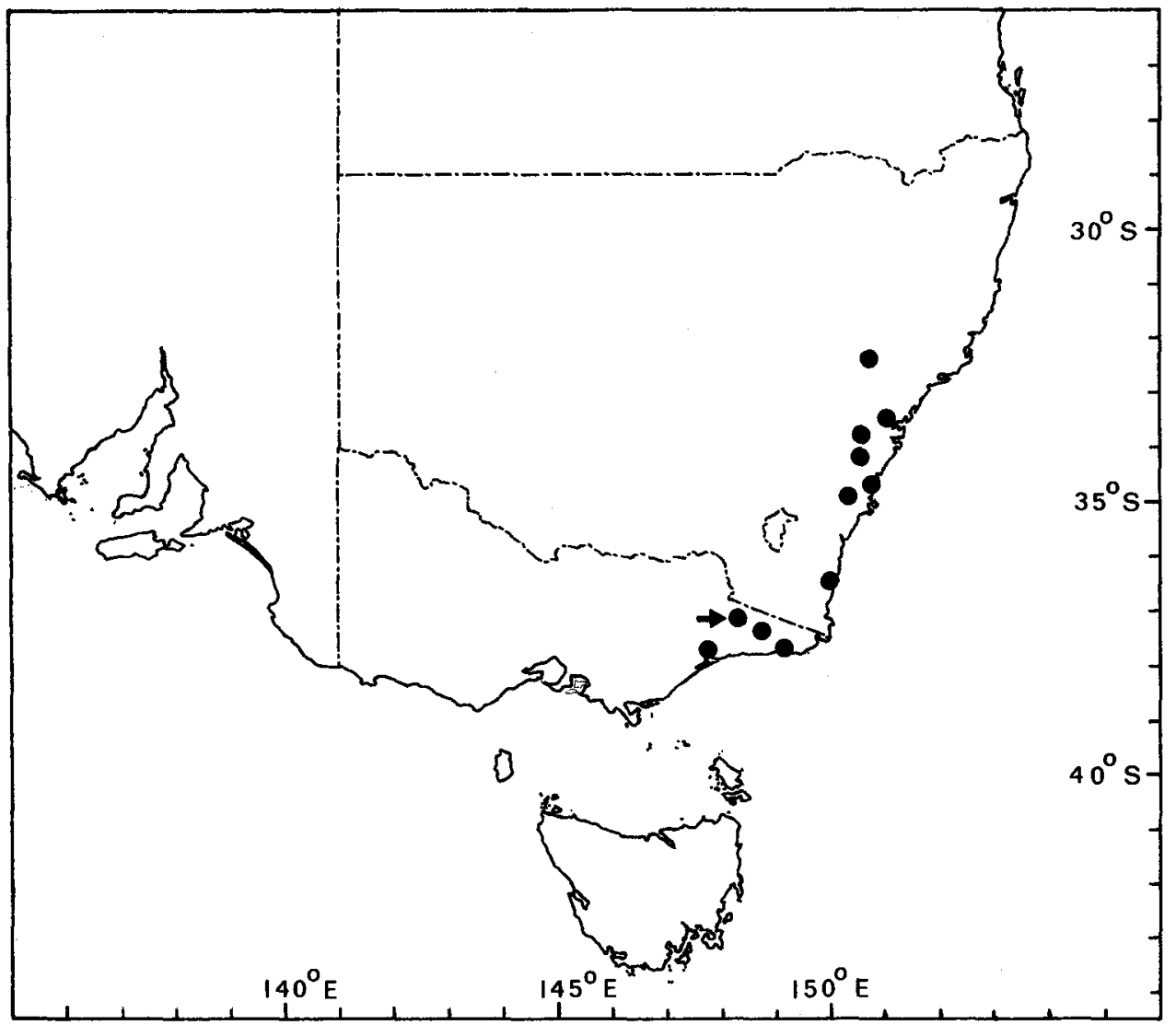

Fig. 6. Distribution of $G$. liratum. Arrow indicates the type locality.

11. Galium liratum Wakefield, Victorian Naturalist 72: 70 incl. figs 2 \& 2a (1955); Beadle et al., Handb. Vasc. Pl. Sydney Distr.: 358 (1963), Fl. Sydney Region: 428 (1972).

HoLOTYPE: [Victoria], Turnback Road, above Little River, near Snowy River, Wakefield 4800, 20.1.1953 (MEL). ISOTYPE: $K$.

Distribution: The species occurs in eastern New South Wales and eastern Victoria, commonly below 200 metres altitude. It favours moist sites in forest or woodland and is usually found growing over or near sandstone or granite rocks. See fig. 6.

Plants ? annual or biennial; root system of primary root, sometimes not strongly developed, nodal roots rare; habit creeping or procumbent. Stems several, (8-) 15-30 $(-80) \mathrm{cm}$ long, retrorsely aculeate to glabrous; internodes $30-50 \mathrm{~mm}$ long. Leaves in whorls of 4 , rarely 5 , obovate to elliptical, (6-) 10-15 (-20) mm long, (1.5-) 2.5-5 (-7) $\mathrm{mm}$ wide, membranaceous; leaf-base conspicuously petiolate; leaf-apex obtusemucronate to broadly acute; leaf-margin slightly recurved to flat; indumentum on upper and lower surfaces sparse, more conspicuous along margin and underside of mid-vein, hairs conical and ascending; venation conspicuous, reticulate; secretory cells absent. Inflorescence sub-pyramidal; ultimate peduncles 1-3 (-5)-flowered, up to 1 $\mathrm{mm}$ long; pedicels $\mathbf{0 . 5}-1.5 \mathrm{~mm}$ long, straight to slightly curved. Flowers inconspicuous, 1.0-1.4 mm diameter, corolla rotate, white. Fruits c. $1.3 \mathrm{~mm}$ long, ribbed, with 5-7 irregular longitudinal ribs.

SPecimens EXamined: New South Wales-Central Coast: Hawkesbury River, near Sydney, Cleland (AD); Euroka Creek, Glenbrook, Whaite 1149, 4.1952 (NSW); Nortons Basin, Coveny NSW 83646, 10.1966 


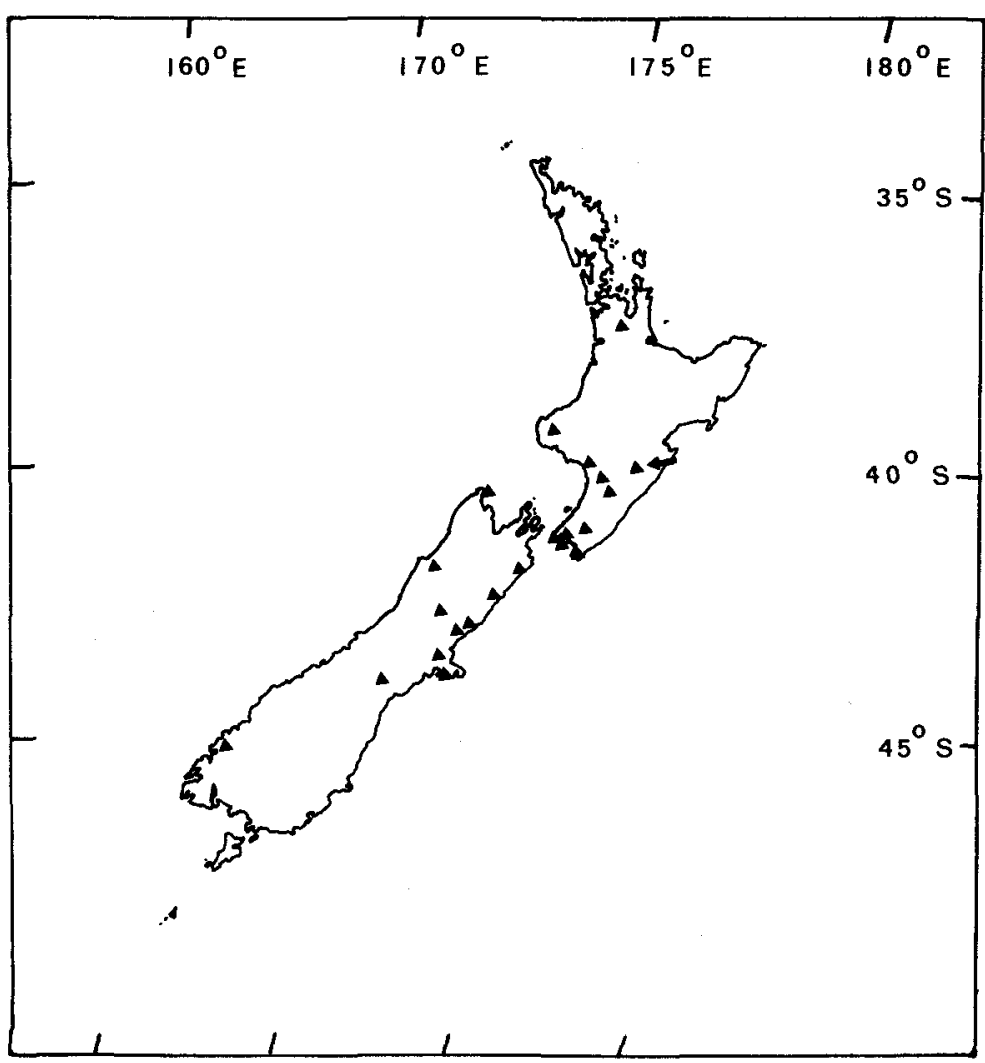

Fig. 7. Distribution of $G$. trilobum. Arrow indicates the type locality.

(NSW); Bents Basin, Woolls (MEL); Nepean River, Douglas Park, McBarron 12365, 4.1966 (NSW); Minnamurra Falls, Hind NSW 84259, 1.1967 (NSW). - South Coast: Yalwal Creek, between Yalwal and Barrier, Rodway 1249, 12.1933 (NSW); Lower Shoalhaven (hills), Bäuerlen 338, 1.1884 (MEL); Mumbulla Mountain, 10 miles [16 km] c. NNE. of Bega, Constable 6804, 5.1966 (NSW).-Central Western Slopes: Denman, Heron NSW 23909, 10.1908 (NSW). VICTORIA: Eastern foot of Mount Ellery, East Gippsland, Wakefield \& Willis, 12.1951 (MEL); Boggy Creek, East Gippsland, Robbins (Beauglehole 7740), c. 1937 (NSW); Sarsfield, 11 miles [18 km] NE. of Bairnsdale, Wakefield 2738, 12.1948 (NSW).

This species is unlikely to be confused with any other. It is characterized by its creeping habit, petiolate leaves usually more than $2.5 \mathrm{~mm}$ wide, very condensed fewflowered cymes with peduncles and pedicels rarely more than $1 \mathrm{~mm}$ long, and its ribbed fruit.

12. Galium trilobum Colenso, Trans. New Zealand Inst. 20: 192 (1888), 'triloba'; Allan, Fl. New Zealand 1: 592 (1961), pro syn.

TYPE: NEW ZEALAND: 'Edge of streamlets in dense shaded woods near Danneverke, County of Waipawa; 1887: W.C.' (protologue), WELT, not seen. IsOTYPE ?: K.

Synonymy: Galium tenuicaule A. Cunn., Ann. Nat. Hist. 2: 205 (1839), nomen illeg., non Krocker (1823), nec Jordan (1852); J.D. Hooker, Fl. Novae-Zelandiae 1: 113 (1852), Handb. New Zealand Fl.: 120 (1864); Kirk Student's Fl. New Zealand: 249 (1899); Cheeseman, Man. New Zealand Fl.: 266 (1906), 879 (1925); Allan, Fl. New Zealand 1: 592 (1961). HoLOTYPE: NEW ZEALAND: In the Kiakatia Wood near the water-fall of Keri Keri. R. Cunningham 1834 (K). 'Damp woods on the Keri Keri and Wangaroa rivers-1834, R. Cunningham.' (protologue).

DiSTRIBUTION: New Zealand, North and South Islands, Stewart Island and Ruapuke Island: favouring damp to swampy situations, e.g. swamp at lake edge; among rocks on edge of fresh water pool; steep damp gully. See fig. 7 . 
Plants perennial ?; root system of nodal roots (primary roots not observed); habit straggling, decumbent or climbing. Stems numerous usually lax, $12-45(-80) \mathrm{cm}$ long, sparingly branched, retrorsely aculeate or sometimes almost smooth; internodes (15-) 25-40 (-50) $\mathrm{mm}$ long. Leaves narrowly obovate to elliptical, sometimes sublinear to spathulate, (4-) 6-12 (-20) $\mathrm{mm}$ long, 1.5-3 (-4) $\mathrm{mm}$ wide, dull green, dark when dry, gradually tapering to a broad petiole-like base; leaf-apex acuminate to acute, sometimes obtuse-mucronate; leaf-margin slightly recurved to flat; leaf-indumentum similar to that of stems; venation obscure, sometimes evident on the lower surface; secretory cells absent. Inflorescence elongate to pyramidal, occupying the upper half to two-thirds of stems, vegetative growth sometimes continuing after flowering; ultimate peduncles (1-) 2-3-flowered, curved, (5-) 8-14 (-25) mm long; pedicels usually curved, (1-) 3-6 (-10) mm long. Flowers $2(-2.5) \mathrm{mm}$ diameter, corolla white, 4- or 3-lobed. Fruits $1-1.5 \mathrm{~mm}$ long, finely ruminate to smooth.

Selected SPECIMENS ( $N$ to $S$; all specimens are from CHR unless otherwise indicated): New ZealandNorth Island: c. 1 mile $[1.5 \mathrm{~km}] \mathrm{N}$ of Mangatarata, Hauraki Plains Co., Mason 7204, 11.1959; Ahukawakawa Swamp, between Egmont and Ponakas Range, Druce 1.1963; Lake Alice near Bulls, Mason 4087, 1.1956; Pauri Lake, S. of Wanganui, Mason 4137, 1.1956; Manawatu Gorge, Allan 1.1932; Near Waiohine River, between Greytown and Cartertown, Healy 53/549, 3.1953; Hutt River, W. side, NW. of upper Hutt, Healy 53/1034, 11.1953; Sinclair Head, Cook Strait, Hamlin 11.1949; Cape Turakirae, Druce 11.1952; Red Rocks, Wellington Coast, Kelly 11.1962; Waitetuma Station, Cape Palliser, Moore 3.1954. - South Island: Para Swamp, Marlborough, Healy 347, 4.1947; Wharf Valley, Marlborough, Mason 2624 \& McQueen, 12.1953; near Leg O'Mutton Lake, W. of Kaikoura, Mason 9262, 2.1962; Burns Swamp, c. $51 / 2$ miles [ $9 \mathrm{~km}$ ] from Kaikoura, Mason 9155, 2.1962; Tidal Creek at bridge on Carters Creek Road, Buller County, Mason 1652 \& Moar, 1.1953; SW. of Spotswood, on Phoebe Road, North Canterbury, Mason 9211, 2.1962; near MacKenzie Domain, Cheviot, Mason 9201, 2.1962; Lake Sumner, Adams 1.1957; View Hill, Oxford, Mitchell 12.1942; Presqu'ile de Banks, Raoul, 1843 (MEL); Lynn Valley, Mount Peel, Allan 4.1919; Lake Katherine, George Sound, Mason 4.1949.

Galium trilobum is characterized by its narrowly obovate to elliptical leaves with no secretory cells, (1-) 2-3-flowered cymes, curved peduncles (5-) 8-14 (-25) mm long and longer than the pedicels, 4- or 3-lobed corolla, and finely ruminate to smooth fruits.

\section{EXCLUDED NAMES}

Galium aparine var. minor Benth., Fl. Austral. 3: $447(1867)=$ G. aparine L., a naturalized alien.

G. geminifolium F. Muell., Trans. Victorian Inst. 1: 127 (1855) and G. umbrosum var. geminifolium (F. Muell.) Moore et Betche, Handb. Fl. New South Wales: $253(1893)=$ Asperula gemella Shaw et Turrill.

G. parisiense var. australe Ewart et White, Proc. Roy. Soc. Victoria n.s. 21: 549 (1909) $=G$. divaricatum Lam., a naturalized alien.

G. perpusillum (Hook. f.) Allan, Fl. New Zealand 1: 592 (1961) = Asperula perpusilla Hook. f. 


\section{ACKNOWLEDGEMENTS}

The generous assistance of Professor F. Ehrendorfer, University of Vienna, is gratefully acknowledged; the inclusion of his name in the author citation of the new names is only a small acknowledgement of his help with the preparation of this paper.

I also wish to thank the Directors of the following herbaria for their generous assistance in providing loans of Galium:-

State Herbarium of South Australia, Adelaide, South Australia (AD);

Botany Department, University of New England, Armidale, New South Wales (NE);

Queensland Herbarium, Brisbane, Queensland (BRI);

Gauba Herbarium, Australian National University, Canberra, Australian Capital Territory (GAUBA);

Herbarium Australiense, Canberra, Australian Capital Territory (CANB);

Canberra Botanic Gardens [now National Botanic Gardens], Canberra, Australian Capital Territory (CBG);

Botany Division, Department of Scientific and Industrial Research, Christchurch, New Zealand (CHR);

Royal Botanic Gardens, Kew, England (K);

British Museum of Natural History, London, England (BM);

John Ray Herbarium, University of Sydney, New South Wales (SYD);

Botanical Museum and Herbarium, Utrecht, Netherlands (U).

Also I wish to acknowledge with many thanks the assistance given and facilities provided by the Curator, Herbarium Australiense, Canberra.

\section{REFERENCES}

Bentham, G. (1867). 'Flora Australiensis.' (London.) Vol. 3.

Black, J.M. (1929). 'Flora of South Australia.' (Adelaide.) Vol. 1.

Colenso, W. (1888). On new phanerogamic plants of New Zealand. Trans. \& Proc. New Zealand Inst. 20: 188-211.

Cunningham, A. (1839). Florae insularum Novae Zelandiae precursor. Ann. Nat. Hist. 2: 205-214.

Ewart, A.J. \& Rees, B. (1913). Contributions to the flora of Australia No. 20. Proc. Roy. Soc. Victoria n.s. 26: 1-11, t. I, II.

Ewart, A.J. (1931). 'Flora of Victoria.' (Melbourne.)

Forster, J.G.A. (1786). 'Florulae insularum australium prodromus.' (Goettingen.)

Hooker, J.D. (1847). Florae Tasmaniae spicilegium. London J. Bot. 6: 461 bis-462 bis.

Hooker, J.D. (1852). 'Florae Novae-Zelandiae. (London.) Vol. 1.

Miquel, F.A.W. (1856). Stirpes Novo-hollandas a Ferd.-Müllero collectas. Nederl. Kruidk. Arch. 4: 113.

Mueller, F.J.H. (1875). 'Fragmenta phytographiae Australiae.' (Melbourne.) Vol. 9.

Wakefield, N.A. (1955). Flora of Victoria: New species and other additions-4. Victorian Naturalist 72: 69-72. 